\title{
FLÓRULA VASCULAR DE LA SIERRA DE CATORCE Y TERRITORIOS ADYACENTES, SAN LUIS POTOSI, MÉXICO
}

\author{
Onésimo González CostillaA ${ }^{1,2}$, Joaquín Giménez de AzCÁrate ${ }^{3}$, José García Pérez ${ }^{1}$ \\ y Juan Rogelio Aguirre Rivera ${ }^{1}$
}
'Universidad Autónoma de San Luis Potosí, Instituto de Investigación de Zonas Desérticas, Altair 200, Fraccionamiento El Llano, Apdo. postal 504, 78377
San Luis Potosí, México.
${ }^{2}$ Universidad Complutense de Madrid, Departamento de Biología Vegetal II, Facultad de Farmacia, Madrid, España. onygc@yahoo.com.mx
${ }^{3}$ Universidad de Santiago de Compostela, Departamento de Botánica, Escuela Politécnica Superior, 27002 Lugo, España.

\section{RESUMEN}

La Sierra de Catorce, localizada en el norte del estado de San Luis Potosí, reúne algunas de las principales cimas del Desierto Chihuahuense cuyas cotas superan los 3000 metros. Ello ha favorecido que la Sierra sea una importante área de diversificación de la flora y las fitocenosis de dicha ecorregión. A partir del estudio fitosociológico de la vegetación del territorio, que se está realizando desde 1999, se ha obtenido un catálogo preliminar de su flora. Hasta el momento la lista de plantas vasculares está conformada por 526 especies y cuatro taxa infraespecíficos, agrupados en 293 géneros y 88 familias. Las familias y géneros mejor representados son Asteraceae, Poaceae, Cactaceae, Fabaceae, Fagaceae y Lamiaceae, así como Quercus, Opuntia, Muhlenbergia, Salvia, Agave, Bouteloua y Dyssodia, respectivamente. Asimismo se señalan los tipos de vegetación representativos del área que albergan los diferentes taxa. Por último, con base en diferentes listas de flora amenazada, se identificaron las especies incluidas en alguna de las categorías reconocidas.

Palabras clave: Desierto Chihuahuense, estudio fitosociológico, flora, flora amenazada, México, San Luis Potosí, Sierra de Catorce.

\section{ABSTRACT}

The Sierra de Catorce located in the north of San Luis Potosí state, shelters some of the most important peaks within the Chihuahuan Desert, whose altitudes reach beyond 
3000 meters. The fact that the Sierra is an important area for flora and plant community diversification in this ecoregion has been favoured by this situation. From a phytosociological study of the territory vegetation, carried out since 1999, we have extracted a preliminary checklist of the flora. Until now the vascular plants checklist is composed by 526 species and four infraespecific taxa, assambled in 293 genera and 88 families. The best represented families and genera are Asteraceae, Poaceae, Cactaceae, Fabaceae, Fagaceae, and Lamiaceae, and Quercus, Opuntia, Muhlenbergia, Salvia, Agave, Bouteloua and Dyssodia respectively. The vegetation types representative of the area which shelter the diferent taxa, are indicated. Finally, from different checklists of threatened flora, the species included in some of the recognised categories are identified

Key words: Chihuahuan Desert, endangered flora, flora, Mexico, phytosociological research, San Luis Potosi, Sierra de Catorce.

\section{INTRODUCCIÓN}

Los primeros trabajos de índole florística efectuados en las zonas áridas y semiáridas del norte de México datan de principios del siglo XIX. Concretamente para el territorio del Altiplano Potosino, J. L. Berlandier, en 1827, fue el pionero en este tipo de estudios (Rzedowski, 1965). Diez años más tarde H. Galeotti realizó una serie de recolectas en las proximidades de la ciudad de San Luis Potosí. Nuevos muestreos fueron efectuados por G. Barroeta hacia el año 1876 en diferentes áreas de la entidad. Destaca también el trabajo de C. Pringle quien, entre 1890 y 1904, recorrió el estado a lo largo de las vías del ferrocarril y recolectó cientos de ejemplares. Otras personas que desarrollaron actividad similar en el Altiplano Potosino durante el siglo XIX fueron Schaffner, Hartweg, Palmer y Palacios. Durante la primera mitad del siglo XX, botánicos como C. L. Lundell, I. M. Johnston, M. C. Johnston, E. Matuda, E. Hernández-X. y H. Bravo aportaron importantes avances relacionados con la sistemática de los principales grupos taxonómicos. Más recientemente, hay que mencionar la prolífica obra de Rzedowski (1955, 1956, 1957, 1959, 1960, 1961, 1962, 1965 y 1978; Rzedowski y Calderón de Rzedowski, 1957), relacionada con diferentes aspectos de la vegetación del estado de San Luis Potosí y, por ende, del Altiplano. En este mismo sentido cabe citar los trabajos de Calderón (1960), Marroquín et al. (1981), Aldrete (1981), Pinkava (1984), González (1991), García-Arévalo y González-Elizondo (1991), Reyes (1992), Villarreal (1994), Reyes et al. (1996) y Zamudio y Pérez (1995), entre otros.

Previamente a que los primeros naturalistas y botánicos recorrieran el Altiplano Mexicano, la Sierra de Catorce, al igual que otros enclaves del centro y norte 
del país, constituyó un centro minero de primera línea a finales del siglo XVIII y principios del XIX. La deforestación, el sobrepastoreo y el crecimiento de la población, vinculados a este auge minero, trajeron consigo una drástica modificación del paisaje vegetal de la Sierra, especialmente en las montañas que rodean el pueblo de Real de Catorce. Las reseñas geohistóricas de Montejano (1993) permiten conocer con certeza cómo era la vegetación circundante. Así, antes del comienzo de la explotación minera, en el último cuarto del siglo XVIII, la Sierra estaba casi deshabitada y cubierta de frondosos bosques, los cuales abastecieron de forma próspera y abundante a las haciendas y minas que se desarrollaron en la zona. Transcurridos 50 años, alrededor de 1825, "no quedaba ni un árbol ni matorral", relatan los cronistas. Por esa época ya no había madera ni en Real ni en Matehuala para abastecer a las minas. Así para 1827, ya se decía que el Real estaba situado en un terreno tan desigual como árido, limitado por las cimas de cerros desnudos, situación que prevalece en la actualidad.

Por ser un área poco estudiada y con el afán de contribuir al conocimiento de la flora del noreste de México, se presenta una lista de las plantas vasculares que crecen en la Sierra de Catorce y territorios adyacentes. No obstante su carácter preliminar, consideramos que servirá de referencia para posteriores trabajos de índole botánica que se efectúen en la región. Un objetivo consecuente del anterior es efectuar un análisis de la participación de los diferentes grupos taxonómicos y el tipo de vegetación donde se encuentran.

\section{CARACTERIZACIÓN DEL ÁREA}

\section{Localización}

El territorio considerado para este estudio incluye tanto la Sierra de Catorce como sus piedemontes y planicies adyacentes. Esta región se localiza en el sector suroriental del Desierto Chihuahuense, al norte del estado de San Luis Potosí. La Sierra constituye una formación montañosa aislada de forma alargada que sirve de eje vertebral del área de estudio. Tiene una dimensión aproximada de $40 \mathrm{~km}$ de longitud, en el sentido N-S, y $35 \mathrm{~km}$ de anchura, en sentido E-W, y está delimitada por las coordenadas $23^{\circ} 16^{\prime} 47^{\prime \prime}-23^{\circ} 53^{\prime} 58^{\prime \prime} \mathrm{N}$, y $100^{\circ} 29^{\prime} 64^{\prime \prime}$ - 101 $01^{\circ} 05^{\prime} 49^{\prime \prime} \mathrm{W}$; sus cumbres, en torno a los 3,000 m s.n.m., se elevan más de 1,500 metros con respecto a las planicies que la bordean. Los límites del territorio considerado están dados por las sierras del Azul (al SE), Coronados (al S), Picachos del Tunalillo (al SW), El Bozal (al W). 
Las anteriores sierras junto con sus planicies adyacentes no fueron consideradas en el mapa propuesto. Otros límites son: la carretera Cedral a San Tiburcio (Zacatecas) al N; la carretera Federal 57 de San Luis Potosí a Saltillo al oriente; la carretera La Bonita-Charcas, al sur; y por último, los lomeríos situados al occidente. Los principales núcleos urbanos, a su vez cabeceras municipales, son: Matehuala, Cedral, Villa de Guadalupe y Real de Catorce (Anónimo, 2002). (Fig. 1).

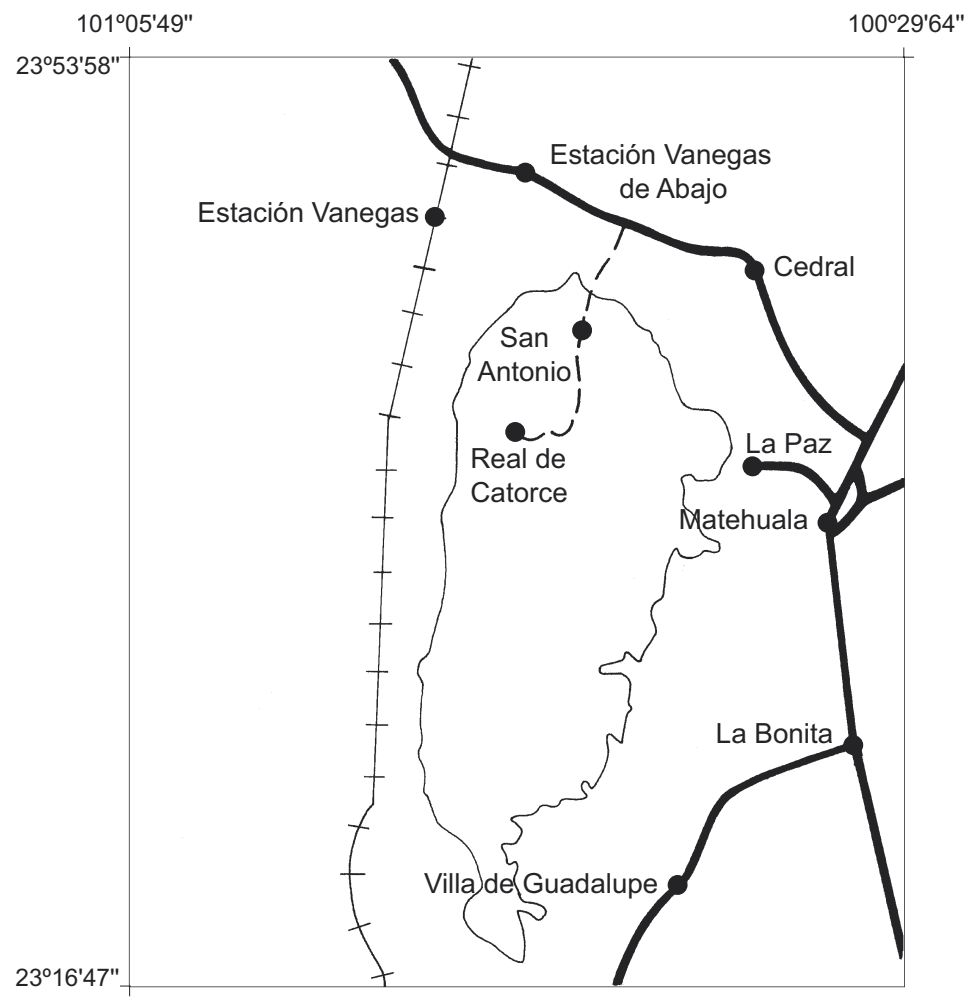

Fig. 1. Localización del área de estudio; el área circundada corresponde a la Sierra de Catorce.

Tras sucesivos decretos estatales fechados en 1994 y 2000, parte del área de estudio situada en la vertiente occidental de la Sierra y en la planicie adyacente (141,000 ha aproximadamente), se encuentra bajo la categoría de Área Natural Protegida, llevando por nombre el de "Reserva Natural y Cultural de Huiricuta". 
Fisiografía y geomorfología

El territorio se integra en el extremo meridional del Altiplano Mexicano (Mullerried, 1944; Rzedowski, 1978), el cual constituye una extensa planicie elevada, con un promedio altitudinal en torno a los $1,700 \mathrm{~m}$, salpicada de serranías orientadas preferentemente en dirección N-S (Rzedowski, 1972); un ejemplo de esta situación alternante de sierra-cuenca lo constituye la región objeto de estudio. En ella la planicie está conformada por sucesivas cuencas sedimentarias de carácter endorréico que bordean la Sierra; su intervalo altitudinal oscila entre los 1,400 m al E de Matehuala y los 2,000 m en el piedemonte occidental de la Sierra. A lo largo del cordal cimero de la Sierra se localizan las principales cumbres: Cerro Grande (3,180 m), Cerro Almagre (3,060 m), Cerro El Barco (3,060 m) y Cerro Puerto del Aire $(3,040 \mathrm{~m})$.

De acuerdo con la clasificación de Raisz (1959), modificada por Medellín (1982), la zona de estudio se incluye en la provincia fisiográfica del Altiplano Central, en la porción suroriental del Desierto Chihuahuense.

Con base en análisis geomorfológicos preliminares efectuados en la zona, se reconoce una diferenciación de procesos y geoformas según la unidad considerada (Morales et al., 2001). En la unidad de la sierra, formada por cumbres y vertientes, dominan los procesos erosivos responsables de una dinámica geomorfológica activa, que ha propiciado un complejo sistema de laderas y valles intermontanos con diferentes formas y pendientes; ello se manifiesta en una marcada asimetría de vertientes, siendo la occidental más escarpada y rectilínea que la oriental, que es más sinuosa y ondulada. A esta intrincada orografía hay que añadir la variabilidad litológica de esta unidad, representada por calizas, areniscas, lutitas, basaltos y conglomerados.

Por el contrario, en las planicies y laderas de contacto dominan los procesos acumulativo-denudativos de materiales procedentes de las partes altas de la Sierra. Las geoformas dominantes en estos ambientes son los abanicos aluviales, los piedemontes, los bolsones, los lomeríos y las mesas basálticas, estos dos últimos considerados como viejas montañas erosionadas (Medellín, 1982).

Clima

Toda la región está sometida a un régimen macroclimático de tipo tropical y de bioclima xérico, caracterizado por la presencia de una corta estación de lluvias en los meses de verano (junio a septiembre) (Rivas-Martínez et al., 1999). Los tipos 
de clima presentes en la región se incluyen mayoritariamente en el grupo de los secos (García, 1981). La posición latitudinal y altitudinal del territorio, su situación geográfica en el interior continental y su régimen tropical, condicionan los rasgos climáticos de la región.

Las características ómbricas vienen dadas por una coincidencia de la época lluviosa con la temporada más cálida del año, por un reparto irregular y dispar de la precipitación tanto en el espacio (de un lugar a otro) como en el tiempo (de un año a otro), por un incremento de la precipitación con la altitud y por un efecto de Foehn de sombra de lluvia en la vertiente occidental de la Sierra (sotavento). Las características térmicas vienen dadas por una oscilación acentuada de la temperatura a lo largo del día, por una evaporación muy intensa favorecida por la fuerte luminosidad, la baja presión y la baja cantidad de humedad atmosférica, y por un gradiente negativo de temperatura y evaporación en relación con la altitud que incrementa la eficiencia de la precipitación.

Todos estos rasgos climáticos, junto con los eventos paleoclimáticos acaecidos durante el Pleistoceno, inciden en la distribución de la flora y de la vegetación a lo largo del territorio y condicionan el escalonamiento altitudinal de los pisos de vegetación (González et al., 2001). De acuerdo con la propuesta de clasificación climática de Köppen (1948), modificada por García (1981), se encuentran en la región de estudio los siguientes grupos:

1. Grupo de climas secos. Presenta lluvias escasas e irregulares principalmente en el verano. Como respuesta, la vegetación potencial se estructura formando diferentes tipos de matorrales dominados por plantas xerófilas. En la zona, los climas secos se presentan en las cotas más bajas vinculadas a los piedemontes; se diferencian los siguientes subtipos:

- Semisecos templados: con lluvias coincidentes con el verano cálido. Se diferencian el $\mathrm{BS}_{1} \mathrm{kw}$ con porcentaje de precipitación invernal entre 5 y 10.2, y el $\mathrm{BS}_{1} \mathrm{kw}\left(\mathrm{x}^{\prime}\right)$ con porcentaje de precipitación invernal mayor de 10.2.

- Seco semicálido $\left(\mathrm{BS}_{0} \mathrm{hx}\right.$ '): con lluvias escasas todo el año, invierno fresco y con porcentaje de precipitación invernal mayor de 18 .

- Seco templado $\left(\mathrm{BS}_{0} \mathrm{kw}\left(\mathrm{x}^{\prime}\right)\right)$ : con lluvias escasas coincidentes con el verano cálido y con porcentaje de precipitación invernal entre 5 y 10.2 .

- Muy seco templado (BWkw): con lluvias muy escasas durante el verano cálido y porcentaje de precipitación invernal entre 5 y 10.2 .

2. Grupo de climas templados. Incluye climas templados subhúmedos, con invierno seco, temperaturas medias anuales entre 12 y $18{ }^{\circ} \mathrm{C}$, y temperaturas del mes más frío entre -3 y $18{ }^{\circ} \mathrm{C}$. La distribución de los climas templados está restringida 
a las zonas medias y altas de la Sierra. Los subtipos reconocidos son el templado subhúmedo $\left[\mathrm{C}\left(\mathrm{w}_{0}\left(\mathrm{x}^{\prime}\right)\right)\right]$, con lluvias de verano y porcentaje de precipitación invernal mayor de 10.2, y el semifrío subhúmedo $\left[\mathrm{C}(\mathrm{E})\left(\mathrm{w}_{1}\right)\right]$ con lluvias de verano y porcentaje de precipitación invernal menor de 5 .

En el Cuadro 1 se presenta información de algunas estaciones meteorológicas localizadas en el territorio de estudio o en su periferia. Además de la situación, se especifica el tipo de clima que corresponde a cada caso.

Cuadro 1. Localización y caracterización climática de algunas estaciones meteorológicas localizadas en el territorio de estudio o en su periferia.

\begin{tabular}{|l|l|c|r|c|c|l|}
\hline \multicolumn{1}{|c|}{ Estación } & Coordenadas & $\begin{array}{c}\text { Altitud } \\
(\mathrm{m})\end{array}$ & Años & $\begin{array}{c}\text { Precipitación } \\
(\mathrm{mm})\end{array}$ & $\begin{array}{c}\text { Temperatura } \\
\text { media anual } \\
\left({ }^{\circ} \mathrm{C}\right)\end{array}$ & \multicolumn{1}{|c|}{ Tipo } \\
\hline Catorce FF CC & $23^{\circ} 41^{\prime} 100^{\circ} 53^{\prime}$ & 1820 & 10 & 269.8 & 18.3 & $\mathrm{BS}_{0} \mathrm{kw}\left(\mathrm{x}^{\prime}\right)$ \\
\hline Cedral & $23^{\circ} 49^{\prime} 100^{\circ} 44^{\prime}$ & 1730 & 20 & 343.9 & 17.5 & $\mathrm{BS}_{0} \mathrm{kx}$ \\
\hline Coyotillos & $23^{\circ} 17^{\prime} 101^{\circ} 12^{\prime}$ & 2200 & 15 & 401.9 & 14.6 & $\mathrm{BS}_{0} \mathrm{kw}$ \\
\hline Charcas & $23^{\circ} 08^{\prime} 101^{\circ} 07^{\prime}$ & 2057 & 26 & 356.2 & 17.2 & $\mathrm{BS}_{0} \mathrm{kw}$ \\
\hline Guaname & $22^{\circ} 52^{\prime} 101^{\circ} 15^{\prime}$ & 1940 & 10 & 462.9 & 16.9 & $\mathrm{BS}_{1} \mathrm{kw}$ \\
\hline La Maroma & $23^{\circ} 28^{\prime} 100^{\circ} 59^{\prime}$ & 1940 & 14 & 325.8 & 17.7 & $\mathrm{BS}_{0} \mathrm{kw}\left(\mathrm{x}^{\prime}\right)$ \\
\hline La Presa & $23^{\circ} 29^{\prime} 100^{\circ} 49^{\prime}$ & 1600 & 15 & 487.1 & 18.6 & $\mathrm{BS}_{0} \mathrm{hw}\left(\mathrm{x}^{\prime}\right)$ \\
\hline La Reforma & $21^{\circ} 45^{\prime} 101^{\circ} 38^{\prime}$ & 2060 & 12 & 330.7 & 16.2 & $\mathrm{BS}_{1} \mathrm{kw}$ \\
\hline Vanegas & $23^{\circ} 53^{\prime} 100^{\circ} 57^{\prime}$ & 1730 & 14 & 302.3 & 16.4 & $\mathrm{BS}_{0} \mathrm{hx} x^{\prime}$ \\
\hline La Victoria & $23^{\circ} 37^{\prime} 101^{\circ} 23^{\prime}$ & 2140 & 9 & 295.0 & 15.6 & $\mathrm{BS}_{0} \mathrm{kw}\left(\mathrm{x}^{\prime}\right)$ \\
\hline
\end{tabular}

Vegetación

La diversidad climática y geomorfológica de la zona, junto con su posición biogeográfica, favorecen la convergencia de fitocenosis diferentes e incluso antitéticas. Aunque se carece de un estudio particular que analice la vegetación del norte de San Luis Potosí, hay que señalar los trabajos de vegetación realizados por Rzedowski $(1957,1961)$, los cuales incluyen en su región de estudio el área aquí tratada, y la contribución de Zamudio y Pérez (1995), quienes describen de forma somera la cubierta vegetal de parte del área que aquí se estudia. Por último cabe mencionar el trabajo realizado por Aldrete (1981) para el noreste de Zacatecas, en el cual se refleja la similitud de los tipos de vegetación de esa región con los presentes en el 
norte de San Luis Potosí. En el aspecto florístico, la zona de estudio alberga una de las mayores concentraciones de cactáceas amenazadas del Desierto Chihuahuense (Hernández y Bárcenas, 1996).

Con base en la clasificación de vegetación propuesta por Rzedowski (1961) para el estado de San Luis Potosí, se describen a continuación las formaciones vegetales (tipos de vegetación) del territorio, con sus respectivos acrónimos empleados en el anexo. Para cada una se adjunta un pequeño comentario de su flora más representativa y de su localización:

Encinar (E): dominado por representantes arbóreos del género Quercus, a los que acompañan muchas de las especies del encinar arbustivo y, ocasionalmente, el pino piñonero (Pinus cembroides). Se localiza preferentemente en las barrancas orientales y meridionales de la Sierra.

Pinar (P): presidido por el pino piñonero (Pinus cembroides var. cembroides) y puntualmente por el pino real (Pinus greggii), aparecen entremezclados en su sotobosque elementos florísticos del encinar. Se localiza preferentemente en la vertiente occidental, parte suroriental y cañadas orientales.

Cedral (C): se corresponde con un matorral escuamifolio dominado por Juniperus erythrocarpa y localizado en un enclave de la vertiente oriental de la Sierra. En las penillanuras de cumbres también se presentan enebrales abiertos de $J$. sabinoides, puros o entremezclados con pinos.

Encinar arbustivo (EA): dominado por especies del género Quercus y con un cortejo florístico entre los que se encuentran Lindleya mespiloides, Arctostaphylos pungens, Comarostaphylis polifolia, Garrya ovata, Garrya laurifolia, Ceanothus greggii, Rhus virens y Eupatorium scorodonioides. Se presenta en las zonas medias y altas de la Sierra.

Matorral submontano (MS): reúne diferentes tipos de comunidades arbustivas dominadas por Helietta parvifolia, Gochnatia hypoleuca, Karwinskia mollis, Opuntia stenopetala, Fraxinus greggii y Ferocactus pilosus, entre otros. Se distribuye a media ladera en la vertiente oriental de la Sierra de Catorce.

Matorral desértico rosetófilo (MDR): con Agave lechuguilla, Agave striata, Dasylirion acrotriche, Hechtia glomerata, Yucca carnerosana, Buddleja marrubiifolia, Salvia ballotaeflora, Fraxinus greggii, Gochnatia hypoleuca, Karwinskia mollis y otras. Incluye también las comunidades edafoxerófilas de Yucca carnerosana. Su distribución está vinculada preferentemente a los lomeríos, piedemontes y exposiciones edafoxerófilas de ladera.

Matorral desértico micrófilo (MDM): formación dominada por Larrea tridentata, Parthenium incanum, Zinnia acerosa, Flourensia cernua, Opuntia imbricata, 
Opuntia leptocaulis, Prosopis laevigata, Fouquieria splendens y otras. Ocasionalmente, en áreas poco deforestadas, emergen de esta formación los izotes (Yucca filifera y $Y$. decipiens).

Matorral crasicaule (MC): formación dominada por especies del género Opuntia (O. leucotricha y O. streptacantha, entre otras), a las que suelen acompañar, Dalea tuberculata, Jatropha dioica, Prosopis laevigata, Forestiera angustifolia, Celtis pallida, Mimosa aculeaticarpa y Yucca carnerosana, entre otras. Se localiza preferentemente en los mogotes y mesas de afloramientos basálticos distribuidos en el suroeste del área de estudio.

Zacatal (Z): Formación herbácea dominada fisionómicamente por gramíneas de bajo porte, como Muhlenbergia repens, Lycurus phleoides, y la presencia de Stipa ichu. Se ubica en las zonas altas de la Sierra sometidas a pastoreo. También se han considerado los pastizales ligados a los sustratos gipsófilos de la planicie nororiental del área de estudio, dominados por Bouteloua chasei, Muhlenbergia purpusii, Dicranocarpus parviflorus, Nama undulatum, Flaveria anomala y Flaveria trinervia, y otras plantas herbáceas.

Otros tipos (OT): Integran los diferentes aprovechamientos y cultivos agrícolas presentes en la zona y su flora arvense asociada. Además se vincula aquí la vegetación y flora viaria y ruderal asociada a zonas con diferentes tipos de perturbación, así como los escasos representantes de la vegetación de rivera. El estudio metódico de estos tipos de vegetación y flora, así como el de otros hábitats restringidos, como los medios acuáticos o rupícolas, se excluyó en el presente trabajo, pero se estima que el incremento del número de especies sea aproximadamente de $20 \%$.

\section{MÉTODOS}

Se integró en una base de datos toda la información florística generada a partir de cerca de 300 inventarios fitosociológicos realizados en la zona de estudio. Para cada tipo de vegetación se anotaron las especies presentes en el área muestreada. Respecto a los métodos relacionados con el enfoque fitosociológico de los estudios de vegetación se consultaron los trabajos de Werger (1974), Westhoff y van der Maarel (1980), y de Gehú y Rivas-Martínez (1981). Paralelamente a la obtención de la información fitosociológica se determinaron los representantes de las especies inventariadas, lo cual se efectuó en el campo y/o laboratorio. El material recolectado e identificado está depositado en el Herbario del Instituto de Investigación de Zonas Desérticas de la Universidad Autónoma de San Luis Potosí (SLPM). 
En el proceso de identificación taxonómica de la flora recolectada se utilizó el Manual de Plantas Vasculares de Texas (Correll y Johnston, 1970). Los rangos taxonómicos empleados (división, clase, familia, género y especie) están basados en las propuestas de Cronquist (1981) y Brummitt (1992). La autoría de las especies se uniformó con base en la obra de Brummitt y Powell (1992), a excepción de las cactáceas que se basó en Hunt (1999).

Un objetivo complementario de este estudio fue identificar aquellos taxones que están incluidos en alguna categoría de protección. Para ello se cotejó la información florística obtenida en este trabajo con la contenida en las listas consultadas, las cuales se incluyen en los siguientes trabajos y marcos legales:

- Convenio sobre el Comercio Internacional de Especies Amenazadas de Fauna y Flora Silvestres (Anónimo, 2001a).

- Norma Oficial Mexicana (Anónimo, 2001b).

- Lista Roja de la IUCN de las Plantas Amenazadas (Walter y Gillett, 1998; Hilton-Taylor, 2000).

- Cactáceas Amenazadas en el Desierto Chihuahuense I y II (Hernández y Bárcenas, 1995 y 1996).

Seguidamente se presenta la estructura y el contenido de la información florística organizada según los siguientes atributos:

- Familia y nombre científico: La ordenación de la lista florística está articulada mediante la secuencia alfabética de familias, géneros y especies. Las familias a su vez se agrupan por clases y divisiones.

- Nombre local: Se presenta en el caso de que la planta tenga una denominación local. Para ello se han seguido tres fuentes de información: el Catálogo de nombres vulgares y científicos de plantas mexicanas (Martínez, 1979), el Catálogo de flora etnobotánica de Matehuala (González, 1991) y las notas recogidas durante las conversaciones con los lugareños.

- Formación vegetal: Para cada taxon de rango específico o inferior se indica la o las formaciones vegetales que reúnen las condiciones ecológicas más en consonancia con los requerimientos del hábitat del taxon en cuestión, de acuerdo con la clasificación indicada anteriormente.

\section{RESULTADOS}

En total se registraron 530 taxones de nivel específico e infraespecífico, agrupados en 293 géneros y 88 familias (Apéndice). La división Magnoliophyta es la 
mejor representada con $96.8 \%$ del total de los taxones registrados, correspondiendo $78.1 \%$ a dicotiledóneas y $18.7 \%$ a monocotiledóneas. Las divisiones Polypodiophyta y Lycopodiophyta integran en su conjunto $1.5 \%$, y Pinophyta $1.7 \%$ de las especies. Las familias mejor representadas son: Asteraceae, Poaceae, Cactaceae, Fabaceae, Fagaceae y Lamiaceae, las cuales, a su vez, reúnen el mayor número de géneros o especies (Cuadro 2). En las cuatro primeras familias se concentra $45.6 \%$ del total de las especies, mientras que en las 84 familias remanentes se encuentra el resto. Estas cifras respaldan lo apuntado por Rzedowski (1991) sobre la importancia de estas familias en la flora del norte del país.

Cuadro 2. Familias con mayor número de géneros y especies en la flora y porcentaje de participación.

\begin{tabular}{|l|c|c|c|}
\hline \multicolumn{1}{|c|}{ Familia } & Número de géneros & Número de especies & \% de especies \\
\hline Asteraceae & 42 & 81 & 15.3 \\
\hline Poaceae & 36 & 68 & 12.9 \\
\hline Cactaceae & 22 & 62 & 11.7 \\
\hline Fabaceae & 17 & 30 & 5.7 \\
\hline Fagaceae & 1 & 17 & 3.2 \\
\hline Lamiaceae & 6 & 15 & 2.8 \\
\hline Rosaceae & 9 & 12 & 2.2 \\
\hline Agavaceae & 2 & 11 & 2.1 \\
\hline Verbenaceae & 6 & 10 & 1.9 \\
\hline Euphorbiaceae & 7 & 10 & 1.9 \\
\hline Total & 148 & 316 & 59.7 \\
\hline
\end{tabular}

Como géneros más representativos destacan, en orden de importancia: Quercus, Opuntia, Muhlenbergia, Salvia, Agave, Bouteloua, Coryphantha, Dyssodia, Eupatorium y Stevia. En el Cuadro 3 se detalla el número de especies incluidas en cada uno de estos géneros.

En cuanto al tipo de formación vegetal que alberga a cada especie en particular se observa una doble tendencia, bien hacia formaciones características de medios xerófilos (matorral desértico micrófilo y matorral desértico rosetófilo, principalmente), o hacia aquellas de ambientes mesofíticos, forestales o preforestales (encinar, pinar, encinar arbustivo y matorral submontano). En cualquier caso las especies exclusivas de una sola formación representan un porcentaje cercano a $10 \%$ 
Cuadro 3. Géneros que incluyen el mayor número de especies.

\begin{tabular}{|l|c|l|c|}
\hline Género & Número de especies & Género & Número de especies \\
\hline Quercus & 17 & Bouteloua & 7 \\
\hline Muhlenbergia & 12 & Coryphantha & 7 \\
\hline Opuntia & 12 & Dyssodia & 7 \\
\hline Salvia & 10 & Eupatorium & 6 \\
\hline Agave & 9 & Stevia & 6 \\
\hline
\end{tabular}

y constituyen especies características de gran valor diagnóstico en el análisis de la vegetación que se está efectuando paralelamente. La mayoría de las especies, debido a su mayor amplitud ecológica, se presenta en dos o más formaciones vegetales próximas entre sí desde el punto de vista estructural, florístico y ecológico.

La heterogeneidad ambiental del territorio es responsable de los distintos tipos de vegetación potencial, que en un gradiente de menor a mayor disponibilidad hídrica, se corresponden con diferentes comunidades de matorrales y bosques. Ello, a priori, favorece el dominio del elemento fanerofítico en el paisaje vegetal del territorio. Sin embargo, la alteración y degradación de la vegetación, ligada fundamentalmente al sobrepastoreo, a la extracción de leña y al avance de la frontera agrícola, ha favorecido la expansión de formaciones dominadas florísticamente por caméfitos; de entre ellos pueden emerger algunos micro y/o mesofanerófitos como Acacia spp., Forestiera angustifolia, Fouquieria splendens, Gochnatia hypoleuca, Lindleya mespiloides, Prosopis spp., Quercus spp. y Yucca spp.

Con el fin de identificar aquella flora presente en el territorio incluida en alguna de las listas de flora amenazada, especificada en el apartado de métodos, se han cotejado éstas con la aquí presentada. Un total de 31 especies están incluidas en alguna de ellas. En el Cuadro 4 se detalla la información recopilada al respecto.

De las 31 especies con algún tipo de protección, 24 tienen preferencia por los matorrales desérticos, mientras que el resto se reparten principalmente entre los diferentes bosques y matorrales y en los zacatales. En cuanto a su adscripción sistemática sobresale el grupo de las cactáceas, el cual constituye casi $75 \%$ de la flora amenazada del área. De lo anterior se deduce que en las planicies y piedemontes que bordean la Sierra, se encuentran las áreas prioritarias para la conservación de la flora del territorio. Pese a que las formaciones vegetales que caracterizan la Sierra sólo reúnen una pequeña proporción de taxones de interés para la conservación, sí albergan una amplia riqueza de taxones de interés biogeográfico, por constituir representantes extremos de su área de distribución o poblaciones relícticas más o 
Cuadro 4. Especies incluidas en alguna categoría de protección. Acrónimos empleados: NOM-059-ECOL: P: en peligro, A: amenazada, Pr: protección especial, E: probablemente extintas en el medio silvestre. UICN: P: en peligro, V: vulnerable, DD: datos deficientes, BR: bajo riesgo, I: indeterminada (categoría previa a 1994), R: rara (categoría previa a 1994). CITES: I: presente en el anexo I, II: presente en el anexo II. Hernández y Bárcenas, 1995: + presente en el apéndice I. * El resto de las cactáceas no incluidas en este cuadro forman parte del Apéndice.

\begin{tabular}{|c|c|c|c|c|}
\hline Especie & NOM-059 ECOL & IUCN & CITES* & $\begin{array}{c}\text { Hernández y } \\
\text { Bárcenas, } 1995\end{array}$ \\
\hline Arbutus xalapensis & & $\mathrm{BR}$ & & \\
\hline Ariocarpus fissuratus & $\mathrm{P}$ & $\mathrm{V}$ & I, II & + \\
\hline Ariocarpus retusus & $\operatorname{Pr}$ & $\mathrm{V}$ & I, II & + \\
\hline Astrophytum capricorne & A & $\mathrm{V}$ & II & + \\
\hline Astrophytum myriostigma & A & $\mathrm{V}$ & II & + \\
\hline Brahea berlandieri & $\operatorname{Pr}$ & & & \\
\hline Bouteloua chasei & & $\mathrm{V}$ & & \\
\hline Cornus disciflora & & $\mathrm{V}$ & & \\
\hline Coryphantha odorata & $\operatorname{Pr}$ & $\mathrm{R}$ & II & \\
\hline Coryphantha poselgeriana & $\mathrm{A}$ & $\mathrm{V}$ & II & + \\
\hline Coryphantha radians & & $\mathrm{R}$ & II & \\
\hline Cupressus arizonica & & $\mathrm{V}$ & & \\
\hline Echinocactus platyacanthus & $\operatorname{Pr}$ & $\mathrm{V}$ & II & + \\
\hline Escobaria dasyacantha & $\operatorname{Pr}$ & $\mathrm{R}$ & II & \\
\hline Ferocactus hamatacanthus & $\operatorname{Pr}$ & $\mathrm{R}$ & II & \\
\hline Ferocactus latispinus & & & II & + \\
\hline Ferocactus pilosus & $\mathrm{P}$ & $\mathrm{V}$ & II & + \\
\hline Leuchtenbergia principis & A & $\mathrm{R}$ & II & + \\
\hline Litsea glaucescens & $\mathrm{P}$ & & & \\
\hline Lophophora williamsii & $\operatorname{Pr}$ & & II & \\
\hline Mammilloydia candida & $\mathrm{A}$ & $\mathrm{I}$ & II & \\
\hline Muhlenbergia purpusii & & $\mathrm{R}$ & & \\
\hline Pinus greggii & & DD & & \\
\hline Plutonopuntia chaffeyi & & $\mathrm{R}$ & II & \\
\hline Quercus depressipes & & DD & & \\
\hline Quercus hintoniorum & & $\mathrm{V}$ & & \\
\hline Sclerocactus uncinatus & & & II & + \\
\hline Thelocactus tulensis & A & $\mathrm{V}$ & I, II & + \\
\hline Turbinicarpus lophophoroides & $\operatorname{Pr}$ & $\mathrm{V}$ & I, II & \\
\hline Turbinicarpus schmiedickeanus & A & $\mathrm{P}$ & I, II & + \\
\hline Turbinicarpus valdezianus & $\operatorname{Pr}$ & & I, II & + \\
\hline
\end{tabular}


menos aisladas. Es el caso de buena parte de la flora nemoral, cuyos ejemplos más evidentes se mencionaron al final del apartado de análisis fitogeográfico.

La conservación y recuperación de la flora emblemática, de sus hábitats y de las formaciones forestales de la Sierra de Catorce y su entorno, deben regir las futuras líneas de actuación vinculadas al manejo de los recursos naturales de la zona, especialmente en el territorio de la Reserva de Huiricuta. El saqueo de cactáceas, la deforestación, el sobrepastoreo, los incendios y la apertura de terracerías, actúan en detrimento del mantenimiento de la biodiversidad en sus diferentes ámbitos y amenazan e hipotecan las alternativas de desarrollo rural sostenido y perdurable.

\section{AGRADECIMIENTOS}

Este estudio ha sido sufragado parcialmente por el proyecto Desierto Chihuahuense de la World Wildlife Foundation (convenio número PP36). Los autores agradecen el apoyo brindado por los herbarios del Instituto de Investigación de Zonas Desérticas de la Universidad Autónoma de San Luis Potosí (IIZD-UASLP) y de la Facultad de Ciencias Biológicas de la Universidad Autónoma de Nuevo León (UANL), así como a Conservación Humana A.C. por su respaldo logístico y organizativo. Se dan las gracias asimismo al Dr. Jorge S. Marroquín y a dos árbitros anónimos por la revisión crítica del documento, a Felicidad García Sánchez (IIZDUASLP) por la determinación de algunos especímenes conflictivos, al Dr. Fernando Zavala Chávez por la revisión de muestras del género Quercus y a la M. en C. Alejandra Fregoso por su apoyo, entusiasmo y compañía durante buena parte del trabajo de campo. Finalmente cabe agradecer también a la Secretaría de Educación Pública (SEP-DGETI) la beca para realizar esta estancia posdoctoral.

\section{LITERATURA CITADA}

Alcaraz, F. 1999. Manual de teoría y práctica de geobotánica. Instituto de Ciencias de la Educación (ICE) de la Universidad de Murcia. Murcia. 401 pp.

Aldrete, M. 1981. Estudios ecológicos del noreste del estado de Zacatecas. Tesis profesional. Universidad Autónoma de Chapingo. Texcoco, Edo. de México. 285 pp.

Anónimo. 2001a. Convención sobre el comercio internacional de especies amenazadas de flora y fauna silvestres. Chatelaine - Geneva. 304 pp. 
Anónimo. 2001b. Norma Oficial Mexicana NOM-059-ECOL-2001. Protección ambientalespecies nativas de México de flora y fauna silvestres-categorías de riesgo y especificaciones para su inclusión, exclusión o cambio-lista de especies en riesgo. Diario Oficial de la Federación. México, D.F.

Anónimo. 2002. Síntesis de información geográfica del estado de San Luis Potosí. Instituto Nacional de Estadística Geografía e Informática. Aguascalientes. 112 pp.

Brummitt, R. K. 1992. Vascular plant families and genera. Royal Botanic Gardens. Kew. 804 pp.

Brummit, R. K. y C. E. Powell. 1992. Authors of plants names. Royal Botanic Gardens. Kew. 732 pp.

Calderón, G. 1960. Notas sobre la flora y vegetación del estado de San Luis Potosí. VII. Vegetación del valle de San Luis Potosí. Acta Cient. Potos. 4: 5-112.

Correll, D. S. y M. C. Johnston. 1970. Manual of the vascular plants of Texas. Texas Research Fundation. Renner. 1881 pp.

Cronquist, A. 1981. An integrated system of classification of flowering plants. Columbia University Press. Nueva York. 262 pp.

García-Arévalo, A. y E. González-Elizondo. 1991. Flora y vegetación de la cima del Cerro Potosí, Nuevo León, México. Acta Bot. Mex. 13: 53-74.

García, E. 1981. Modificaciones al sistema de clasificación climática de Köppen (para adaptarlo a las condiciones de la República Mexicana). Instituto de Geografía. Universidad Nacional Autónoma de México. México, D.F. 256 pp.

Gehú, J. M. y S. Rivas-Martínez. 1981. Notions fondamentales de Phytosociologie. In: Tüxen, R. (ed.). Syntaxonomie. Berichte der Internationalen Symposien der Internationalen Vereinigung für Vegetationskunde. J. Cramer. Vaduz. pp. 5-33.

González, O. 1991. Estudio etnobotánico en el municipio de Matehuala, San Luis Potosí. Ediciones de la Facultad de Ciencias Biológicas. Universidad Autónoma de Nuevo León. Monterrey. 200 pp.

González, O., J. Giménez de Azcárate, J. García y R. Aguirre. 2001. Pisos de vegetación en la Sierra de Catorce. Resúmenes del XV Congreso Mexicano de Botánica. Querétaro.

Hernández, H. y R. Bárcenas. 1995. Endagered cacti in the Chihuahuan Desert: I. Distribution patterns. Conservation Biol. 9(5): 1176-1188.

Hernández, H. y R. Bárcenas. 1996. Endangered cacti in the Chihuahuan Desert: II Biogeography and conservation. Conservation Biol. 10(4): 1200-1209.

Hilton-Taylor, C. 2000. Red list ot threatened species. IUCN (The World Conservation Union). Gland, Suiza y Cambridge. xviii +61 pp.

Hunt, D. 1999. CITES Cactaceae checklist. Remous Limited, Milborne Port. 2a. ed. Royal Botanic Garden. Kew 315 pp.

Köppen, W. 1948. Climatología. Fondo de Cultura Económica. México, D.F. 478 pp.

Marroquín, J. S., G. Borja, R. Velázquez y J. A. de la Cruz. 1981. Estudio ecológicodasonómico de las zonas áridas del norte de México. Pub. Esp. del Instituto Nacional de Investigaciones Forestales. México, D.F. 166 pp.

Martínez, M. 1979. Catálogo de nombres vulgares y científicos de las plantas mexicanas. Fondo de Cultura Económica. México, D.F. 1200 pp. 
Medellín, F. 1982. The Chihuahuan Desert. In: Bender, G. L. (ed.). Reference handbook on the deserts of North America. Greenwood Press. West Port. pp. 321-381.

Montejano, R. 1993. La Purísima Concepción de los Álamos de Catorce. Centro de Estudios Históricos de San Luis Potosí. San Luis Potosí. 123 pp.

Morales, H., A. L. Hernández, J. Giménez de Azcárate y A. Fregoso. 2001. Geomorfología y paisaje de la Reserva Natural y Cultural de Huiricuta (México). Resumen de la VI Reunión Nacional de Geomorfología e International Symposium on Land Degradation and Desertification. México, D.F.

Mullerried, F. 1944. Geología del estado de Nuevo León. An. Inst. Invest. Cient. Universidad de Nuevo León 1: 167-199.

Pinkava, D. J. 1984. Vegetation and flora of the Bolson of Cuatrocienegas region, Coahuila. México: summary, endemism and corrected catalogue. J. Arizona-Nevada Acad. Sci. 19: 23-47.

Raisz, E. 1959. Landforms of México. Escala 1:3,000.000. Map prepared for the Geography Branch of the Office of Naval Research. Cambridge, Mass.

Raunkiaer, C. 1934. The life forms of plants and statistical plant geography. Claredon Press. Oxford. 632 pp.

Reyes, A. 1992. Estudio florístico de la Sierra de Monte Grande, Municipio de Charcas, S.L.P., México. Tesis de Maestría, Colegio de Posgraduados, Texcoco, Edo. de México. 212 pp.

Reyes, A., F. González-Medrano y J. García-Pérez. 1996. Flora vascular de la Sierra de Monte Grande, municipio de Charcas, San Luis Potosí, México. Bol. Soc. Bot. México 58: 31-42.

Rivas-Martínez, S., D. Sánchez-Mata y M. Costa 1999. North American boreal and western temperate forest vegetation (Syntaxonomical synopsis of the potential natural plant communities of North America II ). Itin. Geobot. 12: 5-316.

Rzedowski, J. 1955. Notas sobre la flora y vegetación del Estado de San Luis Potosí. II. Estudios de las diferencias florísticas y ecológicas condicionadas por ciertos tipos de sustrato geológico. Ciencia 15: 141-158.

Rzedowski, J. 1956. Notas sobre la flora y vegetación del Estado de San Luis Potosí III. Vegetación de la región de Guadalcázar. An. Inst. Biol. 27: 169-228.

Rzedowski, J. 1957. La vegetación de las partes áridas de los estados de San Luis Potosí y Zacatecas. Rev. Soc. Mex. Hist. Nat. 8: 49-101.

Rzedowski, J. 1959. Notas sobre la flora y vegetación del estado de San Luis Potosí. VIII. Cinco fanerógamas interesantes del centro y sur del estado. Ciencia 19(4-5): 77-86.

Rzedowski, J. 1960. La vegetación como indicador de rocas en la parte árida del estado de San Luis Potosí. Bol. Soc. Geol. Mex. 23(2): 79-85.

Rzedowski, J. 1961. La vegetación del estado de San Luis Potosí. Tesis de doctorado. Facultad de Ciencias, Universidad Nacional Autónoma de México. México, D.F. 228 pp.

Rzedowski, J. 1962. Contribución a la fitogeografía florística e histórica de México I. Algunas consideraciones acerca del elemento endémico de la flora mexicana. Bol. Soc. Bot. México 27: 52-65.

Rzedowski, J. 1965. Vegetación del estado de San Luis Potosí. Acta Cient. Potos. 5: 2-291. 
Rzedowski, J. 1972. Contribuciones a la fitogeografía florística e histórica de México. III. Algunas tendencias en la distribución geográfica y ecológica de las Compositae mexicanas. Ciencia 27: 123-132.

Rzedowski, J. 1978. Vegetación de México. Editorial Limusa. México, D.F. 432 pp.

Rzedowski, J. 1991. Diversidad y orígenes de la flora fanerogámica de México. Acta Bot. Mex. 14: 3-21.

Rzedowski, J. y G. Calderón de Rzedowski. 1957. Notas sobre la vegetación del estado de San Luis Potosí. IV. Algunas plantas nuevas para la flora del estado. Acta Cient. Potos. 1(2): 199-218.

Villarreal, J. 1994. Flora vascular de la Sierra de la Paila, Coahuila, México. Sida 16(1): 109-138.

Walter, K. S. y H. J. Gillett. 1998. IUCN red list of threatened plants. Compiled by the World Conservation Monitoring Center. IUCN - The World Conservation Union. Gland, Suiza y Cambridge, Inglaterra 862 pp.

Werger, M. J. 1974. On concepts and techniques applied in the Zürich-Montpellier method of vegetation survey. Bothalia (11)3: 309-323.

Westhoff, V. y E. Van der Maarel. 1980. The Braun-Blanquet approach. In: Whittaker, R. H. (ed.). Classification of plant comunities. Dr. W. Junk bv Publishers. La Haya. pp. 287-399.

Zamudio, S. y C. Pérez. 1995. Diagnóstico ecológico y socioeconómico de la región de Real de Catorce o Wirikuta en el Estado de San Luis Potosí. Instituto de Ecología, A.C. Pátzcuaro. Manuscrito inédito. 253 pp. 
Apéndice. Catálogo florístico preliminar, incluyendo los nombres comunes conocidos y la formación vegetal en la que crecen las especies: $\mathrm{C}=$ cedral; $\mathrm{E}=$ encinar; $\mathrm{EA}=$ encinar arbustivo; $\mathrm{MC}=$ matorral crasicaule; $\mathrm{MDM}=$ matorral desértico micrófilo; $\mathrm{MDR}=$ matorral desértico rosetófilo; $\mathrm{MS}=$ matorral submontano; $\mathrm{OT}=$ Otros tipos; $\mathrm{P}=$ pinar; $\mathrm{Z}=$ zacatal.

Familia y especie
PTERIDOPHYTA

\section{ADIANTACEAE}

Adiantum capillus-veneris L.

Cheilantes bonariensis (Willd.) Proctor

Cheilanthes sinuata (Lag. ex Sw.) Domin

Pallaea cordifolia (Sessé \& Moc.) A.R.

Smith

Pellaea sagittata var. cordata (Cav.) A.F.

Tryon

\section{ASPLENIACEAE}

Asplenium resiliens Kunze

POLYPODIACEAE

Polypodium guttatum Maxon

SELAGINELLACEAE

Selaginella pallescens var. acutifolia Stolze

\section{PINOPHYTA}

\section{CUPRESSACEAE}

Cupressus arizonica Greene var. arizonica

Juniperus erythrocarpa Cory

Juniperus sabinoides (Kunth) Nees

\section{EPHEDRACEAE}

Ephedra aspera Engelm. ex S. Watson

Ephedra compacta Rose

Ephedra pedunculata Engelm.

Ephedra trifurca Torr. ex S. Watson

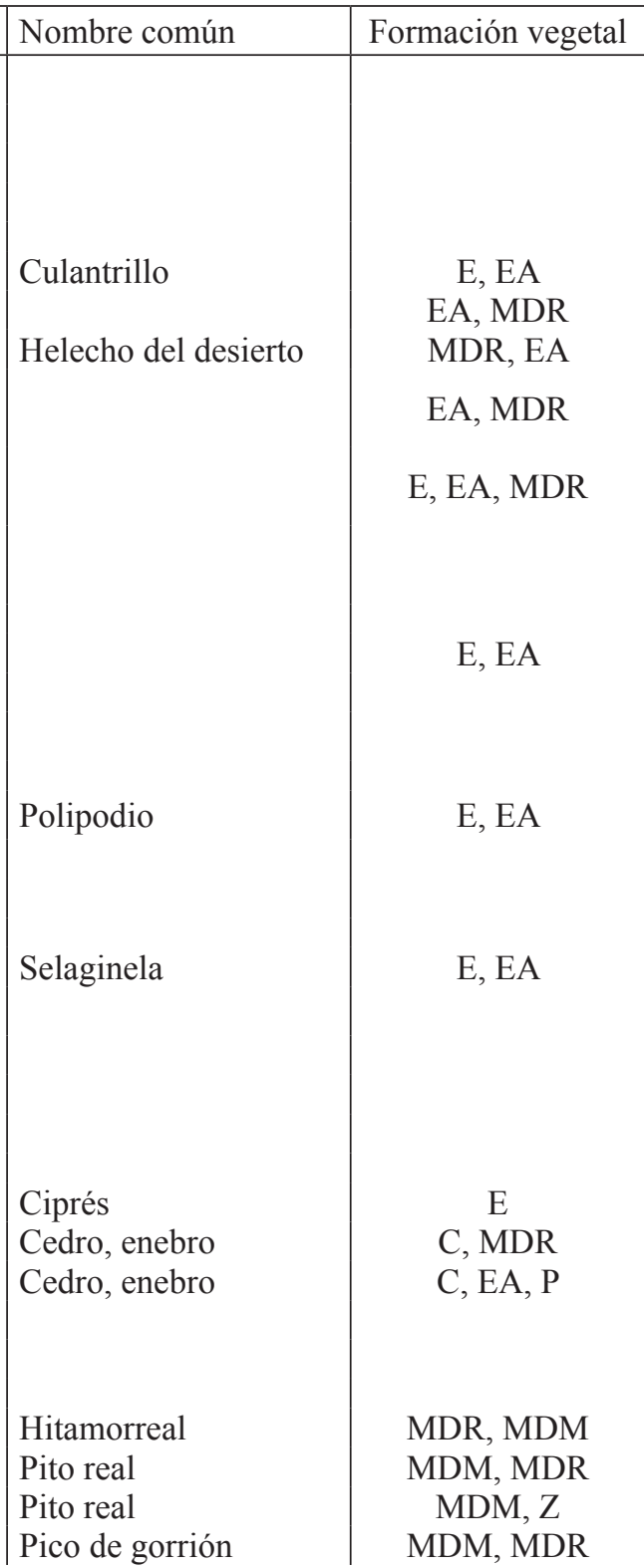


Apéndice. Continuación

Familia y especie
PINACEAE
Pinus cembroides Zucc. var. cembroides
Pinus greggii Engelm.

\section{MAGNOLIOPHYTA}

\section{MAGNOLIOPSIDA}

\section{ACANTHACEAE}

Anisacanthus quadrifidus (Vahl) Nees var. potosinus Henr.

Dyschoriste decumbens (A. Gray) Kuntze

Stenandrium dulce (Cav.) Nees

\section{AMARANTHACEAE}

Alternanthera repens (L.) Kuntze

Amaranthus retroflexus L.

Iresine leptoclada (Hook.f.) Henr. \& Sunb.

Tidestromia lanuginosa (Nutt.) Standl.

\section{ANACARDIACEAE}

Bonetiella anomala (I.M. Johnst.) Rzed.

Rhus aromatica Ait. var. trilobata (Torr. \&

A. Gray) A. Gray

Rhus microphylla Engelm.

Rhus pachyrrhachis Hemsl.

Rhus virens Lindh. ex A. Gray

Schinus molle L.

\section{APOCYNACEAE}

Mandevilla karwinskii (Müll. Arg.) Hemsl. Telosiphonia macrosiphon (Torr.) Henr.

ARALIACEAE

Aralia regeliana March.

\begin{tabular}{|c|c|}
\hline Nombre común & Formación vegetal \\
\hline $\begin{array}{l}\text { Pino piñonero } \\
\text { Pino real }\end{array}$ & $\begin{array}{l}P, E \\
P, E\end{array}$ \\
\hline Cola de gallo & $\begin{array}{c}\text { OT } \\
\text { MDM, MDR } \\
\text { EA, MDR }\end{array}$ \\
\hline $\begin{array}{l}\text { Tianguis } \\
\text { Quelite }\end{array}$ & $\begin{array}{c}\text { OT } \\
\text { OT } \\
\text { MDM } \\
\text { MDR }\end{array}$ \\
\hline $\begin{array}{l}\text { Palo roñoso } \\
\text { Agrito } \\
\text { Agrito } \\
\text { Lantrisco } \\
\text { Lantrisco } \\
\text { Pirul }\end{array}$ & $\begin{array}{c}\text { MS, MDR } \\
\text { P, C, MDR } \\
\text { Z, MDR } \\
\text { E, P, EA } \\
\text { E, P, EA } \\
\text { MC, MDM }\end{array}$ \\
\hline & $\begin{array}{c}\mathrm{C}, \mathrm{MDR} \\
\mathrm{MDR}, \mathrm{MDM}\end{array}$ \\
\hline Aralia & $\mathrm{E}, \mathrm{P}, \mathrm{MS}$ \\
\hline
\end{tabular}


Apéndice. Continuación

\begin{tabular}{|c|c|c|}
\hline Familia y especie & Nombre común & Formación vegetal \\
\hline \multicolumn{3}{|l|}{ ASCLEPIADACEAE } \\
\hline $\begin{array}{l}\text { Asclepias linaria Cav. } \\
\text { Metastelma angustifolium Turcz. }\end{array}$ & $\begin{array}{l}\text { Romerillo, jarilla } \\
\text { texana }\end{array}$ & $\begin{array}{c}\text { MC, MDR } \\
\text { MC }\end{array}$ \\
\hline \multicolumn{3}{|l|}{ ASTERACEAE } \\
\hline $\begin{array}{l}\text { Acourtia nana (A. Gray) Reveal \& R.M. } \\
\text { King }\end{array}$ & & MDM, MDR \\
\hline $\begin{array}{l}\text { Acourtia runcinata (Lag. ex D. Don) } \\
\text { B.L.Turner }\end{array}$ & & MDM, MDR \\
\hline Ageratum corymbosum Zucc. ex Pers. & & E, P, MDR \\
\hline Ambrosia artemisiifolia $\mathrm{L}$. & Altamiz & OT \\
\hline $\begin{array}{l}\text { Ambrosia confertiflora DC. } \\
\text { Aphanosthephus ramosissimus DC. }\end{array}$ & Cola de zorra & $\begin{array}{l}\text { MDM, OT } \\
\text { MDM, MDR }\end{array}$ \\
\hline $\begin{array}{l}\text { Artemisia ludoviciana Nutt. var. mexicana } \\
\text { (Willd.) Fernald }\end{array}$ & Estafiate & MDM, MC \\
\hline Artemisia klotzschiana Besser & & $\mathrm{MC}, \mathrm{MDR}$ \\
\hline Aster subulatus Michx. & & MC, MDR \\
\hline $\begin{array}{l}\text { Dyssodia pentachaeta (DC.) B.L. Rob. } \\
\text { Dyssodia pinnata (Cav.) B.L. Rob. }\end{array}$ & $\begin{array}{l}\text { Parraleña } \\
\text { Rosilla }\end{array}$ & $\begin{array}{l}\text { MDM, MDR, EA } \\
\text { MC, MDR }\end{array}$ \\
\hline $\begin{array}{l}\text { Dyssodia porophyllum var. cancellata } \\
\text { (Cass.) Strother }\end{array}$ & & EA \\
\hline $\begin{array}{l}\text { Dyssodia setifolia (Lag.) B.L. Rob. } \\
\text { Dyssodia tenuifolia (Cass.) Loes. }\end{array}$ & Parraleña & $\begin{array}{l}\text { C, MDR } \\
\text { MC. MDR }\end{array}$ \\
\hline Eupatorium azureum DC. & Gordolobo & MDR, MDM \\
\hline $\begin{array}{l}\text { Eupatorium calophyllum (Greene) B. L. } \\
\text { Rob. }\end{array}$ & & E, C, MDR \\
\hline $\begin{array}{l}\text { Eupatorium espinosarum A. Gray } \\
\text { Eupatorium havanense Kunth }\end{array}$ & Hierba de la mula & $\begin{array}{l}\text { C, EA, MDR } \\
\text { E, P }\end{array}$ \\
\hline $\begin{array}{l}\text { Eupatorium saltillense var. villiferum (B.L. } \\
\text { Rob.) B.L. Turner }\end{array}$ & & $\mathrm{E}$ \\
\hline Eupatorium scorodonioides A. Gray & & $\mathrm{E}, \mathrm{C}, \mathrm{MDR}, \mathrm{EA}$ \\
\hline Flaveria anomala B.L. Rob. & & $\mathrm{Z}, \mathrm{MDM}$ \\
\hline Flaveria oppositifolia (DC.)Rydb. & & $\mathrm{Z}, \mathrm{MDM}$ \\
\hline Flaveria trinervia (Spreng.) C. Mohr & & $\mathrm{Z}, \mathrm{MDM}$ \\
\hline Flourensia cernua DC. & Hojasén & MDM \\
\hline Gochnatia hypoleuca (DC.) A. Gray & Ocotillo & MS, MDR \\
\hline Gutierrezia microcephala (DC.) A. Gray & Escoba de Rosita & MDM \\
\hline $\begin{array}{l}\text { Gutierrezia sarothrae (Pursh) Britton \& } \\
\text { Rusby }\end{array}$ & Escoba de Rosita & MDM \\
\hline
\end{tabular}


Apéndice. Continuación

\begin{tabular}{|c|c|c|}
\hline Familia y especie & Nombre común & Formación vegetal \\
\hline $\begin{array}{l}\text { Gutierrezia texana (DC.) Torr. \& A. Gray } \\
\text { Gymnosperma glutinosum (Spreng.) Less. } \\
\text { Haplopappus spinulosus (Pursh) DC. } \\
\text { Helianthus annuus L. } \\
\text { Jefea brevifolia (A. Gray) J.L. Strother } \\
\text { Jefea gnaphalioides (A. Gray) J.L. Strother } \\
\text { Lessingianthus obtusatus (Less.) H. } \\
\text { Robinson } \\
\text { Parthenium argentatum A. Gray } \\
\text { Parthenium bipinnatifidum (Ortega) Rollins } \\
\text { Parthenium hysterophorus L. } \\
\text { Parthenium incanum Kunth } \\
\text { Piqueria trinervia Cav. } \\
\text { Pinaropappus roseus (Less.) Less. } \\
\text { Porophyllum filiforme Rydb. } \\
\text { Ratibida columnifera (Nutt.) Wooton \& } \\
\text { Standl. } \\
\text { Sanvitalia procumbens Lam. } \\
\text { Senecio praecox (Cav.) DC. var. praecox } \\
\text { Senecio salignus DC. } \\
\text { Solidago scabrida DC. } \\
\text { Stevia eupatoria (Spreng.) Willd. } \\
\text { Stevia lucida Lag. } \\
\text { Stevia micrantha Lag. } \\
\text { Stevia ovata Willd. } \\
\text { Stevia salicifolia Cav. } \\
\text { Stevia serrata Cav. } \\
\text { Taraxacum officinale Wiggers } \\
\text { Tithonia tubiformis (Jacq.) Cass. } \\
\text { Trixis angustifolia DC. } \\
\text { Verbesina encelioides (Cav.) Benth. \& } \\
\text { Hook.f. ex A. Gray } \\
\text { Verbesina longipes Hemsl. } \\
\text { Verbesina rothrockii B.L. Rob. \& Greenm. } \\
\text { Vernonia greggii A. Gray } \\
\text { Vernonia obtusa (Gleason) Blake } \\
\text { Viguiera cordifolia A. Gray } \\
\text { Viguiera dentata (Cav.) Spreng. } \\
\text { Viguiera greggii (A. Gray) S.F. Blake } \\
\text { Viguiera stenoloba S.F. Blake } \\
\text { Zaluzania triloba (Ortega) Pers. } \\
\text { Zinnia acerosa (DC.) A. Gray } \\
\text { Zinnia juniperifolia (DC.) A. Gray }\end{array}$ & $\begin{array}{l}\text { Escoba de Rosita } \\
\text { Jarilla } \\
\text { Hierba de la víbora } \\
\text { Polocote, girasol } \\
\text { Malacatillo }\end{array}$ & $\begin{array}{c}\text { MDM } \\
\text { MDM, MDR } \\
\text { MDM, MDR } \\
\text { OT } \\
\text { MDR } \\
\text { MDR } \\
\text { P, E } \\
\text { MDR, MDM } \\
\text { OT } \\
\text { OT } \\
\text { MDM, MDR } \\
\text { Z, OT } \\
\text { P, MDR } \\
\text { EA, MDR } \\
\text { Z } \\
\text { OT } \\
\text { MC, E, EA } \\
\text { EA, C } \\
\text { OT } \\
\text { E, EA } \\
\text { P, MDM } \\
\text { P, MDM } \\
\text { P, MDM } \\
\text { Z, EA } \\
\text { P, MDM } \\
\text { OT } \\
\text { MDM } \\
\text { MDR, MC } \\
\text { MC, MDM } \\
\text { MC } \\
\text { MDR } \\
\text { P, E } \\
\text { P, E } \\
\text { EDR, MDM } \\
\text { MDM , MDM } \\
\text { MDR, MDM } \\
\text { MDM } \\
\text { MDM } \\
\text { MDR, MC }\end{array}$ \\
\hline
\end{tabular}


Apéndice. Continuación

\begin{tabular}{|c|c|c|}
\hline Familia y especie & Nombre común & Formación vegetal \\
\hline BERBERIDACEAE & & \\
\hline $\begin{array}{l}\text { Berberis pinifolia (Lundell) C.H. Muller } \\
\text { Berberis trifoliolata Moric. }\end{array}$ & $\begin{array}{l}\text { Agrito } \\
\text { Agrito }\end{array}$ & $\begin{array}{l}\text { MC, } \mathrm{MDR} \\
\mathrm{MDR}, \mathrm{MC}\end{array}$ \\
\hline BIGNONIACEAE & & \\
\hline $\begin{array}{l}\text { Chilopsis linearis (Cav.) Sweet var. linearis } \\
\text { Tecoma stans (L.) Kunth var. stans }\end{array}$ & Tronadora & $\begin{array}{l}\text { MDR, MS } \\
\text { MDR, MS }\end{array}$ \\
\hline BORAGINACEAE & & \\
\hline $\begin{array}{l}\text { Antiphytum heliotropioides DC. } \\
\text { Antiphytum parryi S. Watson } \\
\text { Tiquilia canescens (DC.) A.T. Richardson }\end{array}$ & $\begin{array}{l}\text { Zarzaparrilla } \\
\text { Hierba de la hormiga }\end{array}$ & $\begin{array}{l}\text { MDR, EA } \\
\text { MS, MDR } \\
\text { MDM }\end{array}$ \\
\hline BRASSICACEAE & & \\
\hline $\begin{array}{l}\text { Halimolobos lasioloba (Link) O.E. Schulz } \\
\text { Halimolobos polysperma (Fourn.) O.E. } \\
\text { Schulz } \\
\text { Halimolobos rigida Rollins } \\
\text { Lesquerella fendleri (A. Gray) S. Watson } \\
\text { Sisymbrium irio L. }\end{array}$ & Colesilla & $\begin{array}{c}\text { OT } \\
\text { E, EA } \\
\text { EA, OT } \\
\text { MDR, MDM } \\
\text { OT }\end{array}$ \\
\hline BURSERACEAE & & \\
\hline Bursera fagaroides (Kunth) Engl. & & MDR, MS \\
\hline CACTACEAE & & \\
\hline $\begin{array}{l}\text { Ariocarpus bravoanus ssp. hintonii (Stuppy } \\
\text { \& N.P. Taylor) F. Anderson \& W. A. Fitz } \\
\text { Maurice }\end{array}$ & & MDR, MDM \\
\hline Ariocarpus retusus Scheidw. & Chaute & MDR, MS \\
\hline $\begin{array}{l}\text { Astrophytum capricorne (Dietr.) Britton \& } \\
\text { Rose } \\
\text { Astrophytum myriostigma Lemaire ssp. } \\
\text { myriostigma }\end{array}$ & Chaute & $\begin{array}{l}\text { MDR, MDM } \\
\text { MDM }\end{array}$ \\
\hline $\begin{array}{l}\text { Coryphantha compacta (Engelm.) Britton } \\
\text { \& Rose } \\
\text { Coryphantha echinoidea (Quehl.) Britton } \\
\text { \& Rose }\end{array}$ & Biznaga & $\begin{array}{c}\text { MDM } \\
\text { MDR, MDM }\end{array}$ \\
\hline
\end{tabular}


Apéndice. Continuación

\begin{tabular}{|c|c|c|}
\hline Familia y especie & Nombre común & Formación vegetal \\
\hline $\begin{array}{l}\text { Coryphantha glanduligera (Otto ex Dietr.) } \\
\text { Lem. }\end{array}$ & Biznaga & MDM, MDR \\
\hline Coryphantha macromeris (Engelm.) Lem. & & MDR, MDM \\
\hline $\begin{array}{l}\text { Coryphantha odorata Boedeker } \\
\text { Coryphantha poselgeriana (Districh) }\end{array}$ & Biznaga & MDR, MDM \\
\hline Britton \& Rose ssp. poselgeriana & & MDM, MDR \\
\hline Coryphantha radians (DC.) Britton \& Rose & & MDR \\
\hline $\begin{array}{l}\text { Cylindropuntia imbricata (Haw.) F.M. } \\
\text { Kunth }\end{array}$ & Coyonoixtle & MDM, MC, MDM \\
\hline $\begin{array}{l}\text { Echinocactus platyacanthus Link \& Otto f. } \\
\text { grandis (Rose) H. Bravo }\end{array}$ & Biznaga gigante & MDR, EA \\
\hline $\begin{array}{l}\text { Echinocactus platyacanthus Link \& Otto f. } \\
\text { viznaga (Hook.) H. Bravo }\end{array}$ & Biznaga gigante & MDR, EA \\
\hline $\begin{array}{l}\text { Echinocereus cinerascens (DC.) Lem. var. } \\
\text { septentrionalis N.P. Taylor }\end{array}$ & Alicoche & MDM, MDR \\
\hline $\begin{array}{l}\text { Echinocereus cinerascens ssp. tulensis } \\
\text { (Bravo) Taylor }\end{array}$ & Alicoche & MDR, MS \\
\hline $\begin{array}{l}\text { Echinocereus merkeri Hildman ex } \\
\text { Schumann }\end{array}$ & Alicoche & MDM, MDR \\
\hline $\begin{array}{l}\text { Echinocereus pectinatus (Scheidw.) } \\
\text { Engelm. ssp. pectinatus }\end{array}$ & Biznaga & MDR, MDM \\
\hline Echinocereus pentalophus (DC.) Lem. & Alicoche & MDR, MS \\
\hline Echinocereus stramineus (Engelm.) Seitz & Alicoche verde & MDM \\
\hline $\begin{array}{l}\text { Echinocereus weinbergii (Weingart) N.P. } \\
\text { Taylor }\end{array}$ & & MDM \\
\hline $\begin{array}{l}\text { Epithelantha micromeris (Engelm.) Weber } \\
\text { f. elongata (Backeb.) Bravo }\end{array}$ & & MDR \\
\hline $\begin{array}{l}\text { Escobaria dasyacantha (Engelm.) Britton } \\
\text { \& Rose }\end{array}$ & & MDR, MS \\
\hline $\begin{array}{l}\text { Ferocactus hamatacanthus (Muehlenpf.) } \\
\text { Britton \& Rose }\end{array}$ & Uña de gavilán & MDM \\
\hline Ferocactus histrix (DC.) Linds. & & MC, MDR \\
\hline Ferocactus horizontalonius Lem. & Mancacaballos & MDM \\
\hline $\begin{array}{l}\text { Ferocactus latispinus (Haw.) Britton \& } \\
\text { Rose }\end{array}$ & Biznaga & MDM, MDR \\
\hline $\begin{array}{l}\text { Ferocactus pilosus (Galeotti ex Salm-Dyck) } \\
\text { Werderm. }\end{array}$ & Biznaga colorada & MDR, EA, MS \\
\hline Leuchtenbergia principis Hook. & & MDR, MDM \\
\hline $\begin{array}{l}\text { Lophophora williamsii var. echinata } \\
\text { (Croizat) Bravo }\end{array}$ & Peyote & MDM \\
\hline $\begin{array}{l}\text { Lophophora williamsii var. williamsii Lem. } \\
\text { ex Salm-Dyck }\end{array}$ & Peyote & MDM \\
\hline
\end{tabular}


Apéndice. Continuación

\begin{tabular}{|c|c|c|}
\hline Familia y especie & Nombre común & Formación vegetal \\
\hline Mammillaria compressa DC. & Biznaga chilera & MDM \\
\hline Mammillaria heyderi Muehlenpf. & & MDM \\
\hline Mammillaria formosa Galeotti ex Scheidw. & Biznaga & MDM, MDR \\
\hline Mammillaria pottsii Scheer. & Biznaga & MDM, MDR \\
\hline Mammilloydia candida (Scheidw.) Buxb. & Viejito & MDR, MS \\
\hline $\begin{array}{l}\text { Myrtillocactus geometrizans (Mart.) } \\
\text { Console }\end{array}$ & Garambullo & MDM \\
\hline Neolloydia conoidea (DC.) Britton \& Rose & & MDR, MS \\
\hline Neolloydia matehualensis Backeb. & & MDR, MS \\
\hline Opuntia engelmannii var. cuija Griffiths & Nopal cuija & MDM \\
\hline Opuntia ficus-indica (L.) Mill. & Nopal pelón & OT \\
\hline Opuntia kleiniae DC. & Tasajillo & MDM, Z \\
\hline Opuntia imbricata (Haw.) DC. & Coyonoistle & MDM, MC \\
\hline Opuntia leptocaulis DC. & Tasajillo & MDM, Z \\
\hline Opuntia leucotricha DC. & Nopal duraznillo & $\mathrm{MC}, \mathrm{MDM}$ \\
\hline $\begin{array}{l}\text { Opuntia microdasys (Lehm.) Pfeiff. var. } \\
\text { pallida Hort. }\end{array}$ & Nopal cegador & $\mathrm{MC}, \mathrm{MDM}$ \\
\hline Opuntia rastrera F.A.C. Weber & Nopal rastrero & MDM \\
\hline Opuntia robusta $\mathrm{H}$. Wendl. & Nopal tapón & MC, MDM, E \\
\hline Opuntia stenopetala Engelm. & Nopal tuna colorada & MDR, MS, EA \\
\hline Opuntia streptacantha Lem. & Nopal cardón & MDM, MC \\
\hline Opuntia tunicata (Lehm.) Link \& Otto & Clavellina & MDM \\
\hline Opuntia villis Rose & Clavellina & MDM, Z \\
\hline $\begin{array}{l}\text { Pachycereus marginatus (DC.) Britton \& } \\
\text { Rose }\end{array}$ & Órgano & OT \\
\hline Pelecyphora aselliformis C.G. Ehrenberg & & MDR, MDM \\
\hline Pelecyphora pulcherrima Sabatini & & MDR, MDM \\
\hline $\begin{array}{l}\text { Plutonopuntia chaffeyi (Britton \& Rose) } \\
\text { P.V. Heath. }\end{array}$ & Zacasil & E, EA \\
\hline Sclerocactus uncinatus (Galeotti) Taylor & Uña de gavilán & MDM, MDR \\
\hline Stenocactus crispatus (DC.) Berger ex Hill & Biznaguita & MC, MDR, EA \\
\hline $\begin{array}{l}\text { Stenocactus dichroacanthus (Mart. ex } \\
\text { Pfeiffl.) A. Berger ex Backeb. }\end{array}$ & Biznaga & $\mathrm{MC}, \mathrm{E}, \mathrm{EA}$ \\
\hline $\begin{array}{l}\text { Thelocactus bicolor (Galeotti ex Pfeiff.) } \\
\text { Britton \& Rose ssp. bicolor }\end{array}$ & & MDM, MDR \\
\hline $\begin{array}{l}\text { Thelocactus conothelos (Regel \& Klein) } \\
\text { Backeb. \& Knuth }\end{array}$ & & MDM, MDR, MS \\
\hline $\begin{array}{l}\text { Thelocactus hexaedrophorus (Lem.) Britton } \\
\text { \& Rose }\end{array}$ & & MDM, Z \\
\hline $\begin{array}{l}\text { Thelocactus rinconensis (Pos.) Britton \& } \\
\text { Rose }\end{array}$ & & MDR, MDM \\
\hline
\end{tabular}


Apéndice. Continuación

\begin{tabular}{|c|c|c|}
\hline Familia y especie & Nombre común & Formación vegetal \\
\hline Thelocactus tulensis (Pos.) Britton \& Rose & & MDR, MS \\
\hline $\begin{array}{l}\text { Turbinicarpus gielsderfianus (Werder.) V. } \\
\text { John \& Riba }\end{array}$ & & MDM, MDR \\
\hline Turbinicarpus laui Glass \& Foster & & MDR, MDM \\
\hline $\begin{array}{l}\text { Turbinicarpus lophophorioides (Werderm.) } \\
\text { Buxb. \& Backeb. }\end{array}$ & & MDM \\
\hline $\begin{array}{l}\text { Turbinicarpus macrochele (Werderm.) } \\
\text { Buxb. \& Backeb. }\end{array}$ & & MDM, MDR \\
\hline $\begin{array}{l}\text { Turbinicarpus macrochele var. schwarzii } \\
\text { (Surly) Kladiva }\end{array}$ & & MDM, MDR \\
\hline $\begin{array}{l}\text { Turbinicarpus schmiedickeanus (Boedeker) } \\
\text { Buxb. \& Backeb. }\end{array}$ & & MDR, MS \\
\hline $\begin{array}{l}\text { Turbinicarpus valdezianus (H. Moeller) } \\
\text { Glass \& M.B. Foster }\end{array}$ & & MDR \\
\hline CAPRIFOLIACEAE & & \\
\hline Lonicera pilosa (Kunth) Willd. & & EA \\
\hline CARYOPHYLLACEAE & & \\
\hline Arenaria lycopodioides Willd. ex Schlecht. & & MDR, EA \\
\hline Drymaria anomala $\mathrm{S}$. Watson & & EA, MDR \\
\hline $\begin{array}{l}\text { Drymaria arenarioides Willd. ex Roem. \& } \\
\text { Schult }\end{array}$ & & $\mathrm{MC}, \mathrm{Z}$ \\
\hline CELASTRACEAE & & \\
\hline $\begin{array}{l}\text { Acanthothamnus aphyllus (Schltdl.) Standl. } \\
\text { Maytenus phyllanthoides Benth. } \\
\text { Mortonia greggii A. Gray } \\
\text { Orthosphenia mexicana Standl. }\end{array}$ & $\begin{array}{l}\text { Granadilla } \\
\text { Afinador } \\
\text { Cola de zorra }\end{array}$ & $\begin{array}{l}\text { MC, MDM } \\
\text { MDM } \\
\text { MDM, MDR } \\
\text { P, MS, EA }\end{array}$ \\
\hline CHENOPODIACEAE & & \\
\hline $\begin{array}{l}\text { Atriplex canescens (Pursh) Nutt. } \\
\text { Atriplex obovata Moq. } \\
\text { Chenopodium blitoides Lej. } \\
\text { Salsola tragus L. } \\
\text { Teloxys graveolens (Willd.) W.A. Weber }\end{array}$ & $\begin{array}{l}\text { Costilla de vaca } \\
\text { Saladilla } \\
\text { Quelite de cochino } \\
\text { Rodadora } \\
\text { Epazote de zorrillo }\end{array}$ & $\begin{array}{c}\text { MDM, OT } \\
\text { MDM, OT } \\
\text { OT } \\
\text { OT } \\
\text { MC, MDR, EA }\end{array}$ \\
\hline
\end{tabular}


Apéndice. Continuación

\begin{tabular}{|c|c|c|}
\hline Familia y especie & Nombre común & Formación vegetal \\
\hline CISTACEAE & & \\
\hline Helianthemum glomeratum Lag. & Juanita & $\mathrm{P}, \mathrm{C}$ \\
\hline CONVOLVULACEAE & & \\
\hline Cuscuta applanata Engelm. & Fideo & $\mathrm{E}$ \\
\hline Cuscuta glabrior (Engelm.) Yunck. & Fideo & $\mathrm{E}$ \\
\hline $\begin{array}{l}\text { Dichondra argentea Humb. \& Bonpl. ex } \\
\text { Willd }\end{array}$ & Oreja de ratón & MDR, MDM, P \\
\hline Ipomoea purpurea (L.) Roth & Corrihuela & $\mathrm{E}$ \\
\hline Ipomoea sescossiana Baill. & Enredadera & $\mathrm{E}$ \\
\hline CORNACEAE & & \\
\hline Cornus disciflora Moc. \& Sessé ex DC. & & E, P, EA \\
\hline CRASSULACEAE & & \\
\hline Echeveria coccinea (Cav.) DC. & & MC, MDR \\
\hline Echeveria mucronata Schltdl. & & $\mathrm{E}, \mathrm{P}$ \\
\hline Echeveria secunda Booth & & $\mathrm{MC}$ \\
\hline $\begin{array}{l}\text { Kalanchoe coccinea var. blossfeldiana } \\
\text { (Poelln.) P. Bolteau }\end{array}$ & & MDM \\
\hline Sedum catorce G.L. Nelson & & $\mathrm{MC}, \mathrm{MDR}$ \\
\hline CUCURBITACEAE & & \\
\hline Apodanthera undulata A. Gray & Melón loco & MDM, Z \\
\hline Cucurbita foetidissima Kunth & Calabacilla loca & OT \\
\hline Cuсurbita pepo L. & Calabaza & OT \\
\hline Ibervillea tenuisecta (A. Gray) Small & & $\mathrm{MC}, \mathrm{MDM}$ \\
\hline ERICACEAE & & \\
\hline Arctostaphylos pungens Kunth & Pingüica & $\mathrm{P}, \mathrm{E}, \mathrm{EA}$ \\
\hline Arbutus xalapensis Kunth & Madroño & E, EA \\
\hline $\begin{array}{l}\text { Comarostaphylis polifolia (Kunth) Zucc. ex } \\
\text { Klotzsch ssp. polifolia }\end{array}$ & Macuate & $\mathrm{E}, \mathrm{P}, \mathrm{EA}$ \\
\hline EUPHORBIACEAE & & \\
\hline Acalypha monostachya Cav. & & MDM, MDR \\
\hline
\end{tabular}


Apéndice. Continuación

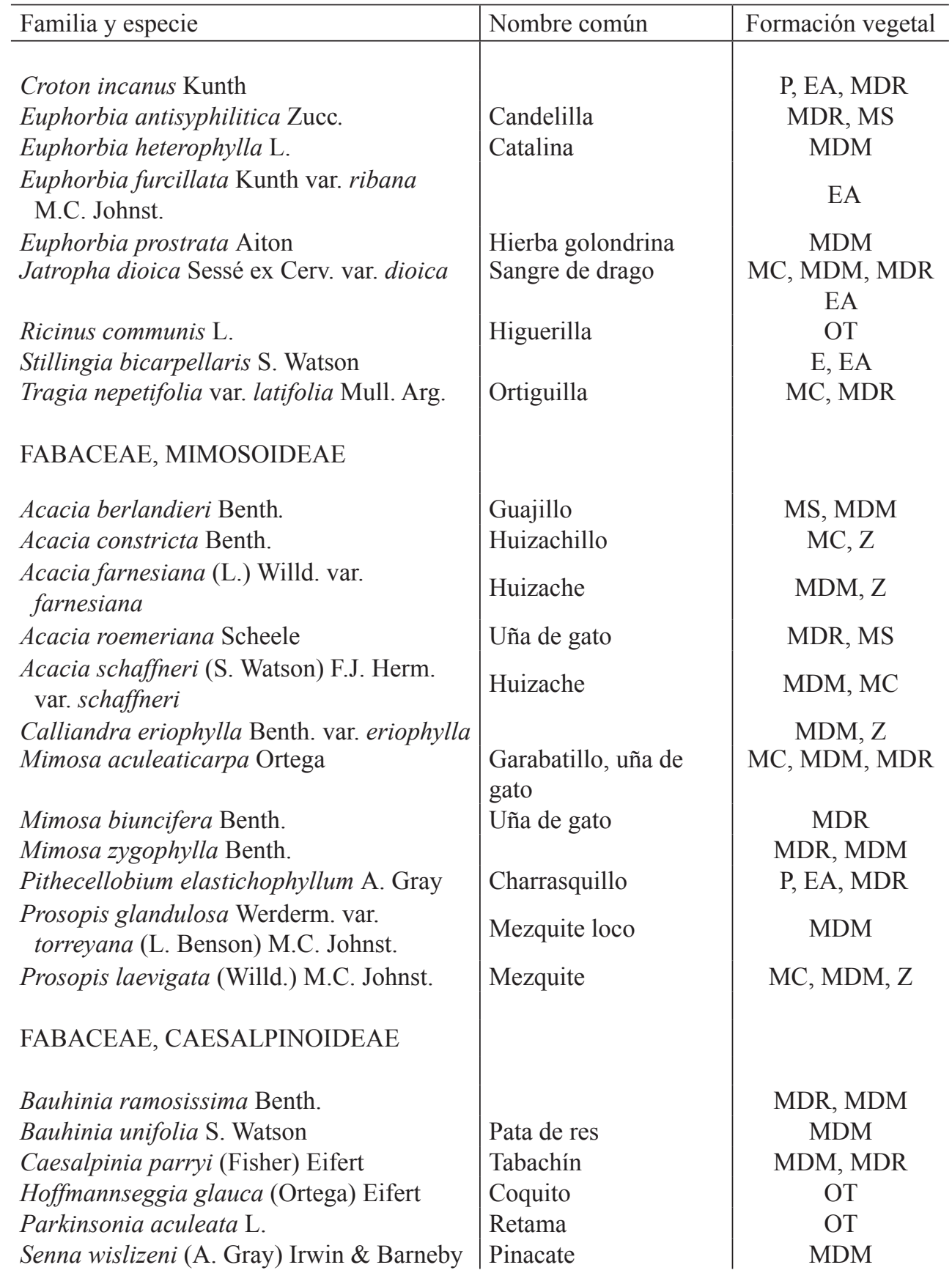


Apéndice. Continuación

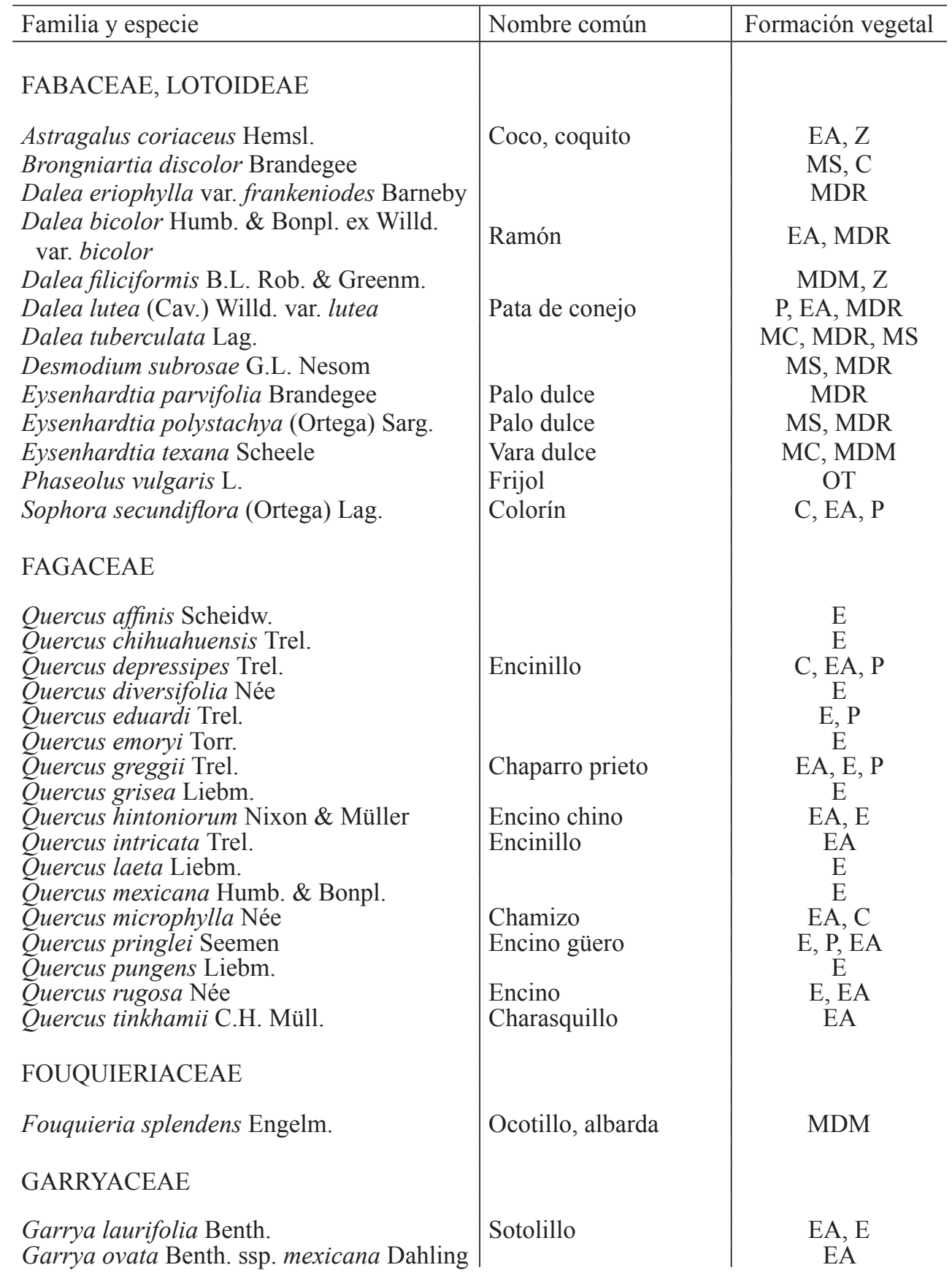


Apéndice. Continuación

\begin{tabular}{|c|c|c|}
\hline Familia y especie & Nombre común & Formación vegetal \\
\hline GERANIACEAE & & \\
\hline Erodium cicutarum ( L.) L' Hér. & & $\mathrm{P}, \mathrm{EA}, \mathrm{Z}$ \\
\hline HYDROPHYLLACEAE & & \\
\hline $\begin{array}{l}\text { Nama palmeri A. Gray ex Hemsl. } \\
\text { Nama undulatum Kunth }\end{array}$ & $\begin{array}{l}\text { Ventosidad } \\
\text { Ventosidad }\end{array}$ & $\begin{array}{c}\text { MDR } \\
\mathrm{Z}, \mathrm{MDM}\end{array}$ \\
\hline KOEBERLINIACEAE & & \\
\hline Koeberlinia spinosa Zucc. & Junco & MDM, Z \\
\hline KRAMERIACEAE & & \\
\hline $\begin{array}{l}\text { Krameria cytisoides Cav. } \\
\text { Krameria navae Rzed. }\end{array}$ & & $\begin{array}{l}\text { MDR, MDM, P } \\
\text { MDR, MS }\end{array}$ \\
\hline LAMIACEAE (LABIATAE) & & \\
\hline $\begin{array}{l}\text { Hedeoma drummondii Benth. } \\
\text { Marrubium vulgare L. } \\
\text { Poliomintha longiflora A. Gray } \\
\text { Salvia ballotiflora Benth. } \\
\text { Salvia chamaedryoides Cav. } \\
\text { Salvia greggii A. Gray } \\
\text { Salvia keerlii Benth. } \\
\text { Salvia lycioides A. Gray } \\
\text { Salvia mexicana } \text { L. } \\
\text { Salvia microphylla Kunth } \\
\text { Salvia reflexa Hornem. } \\
\text { Salvia regla } \text { Cav. } \\
\text { Salvia tiliifolia } \text { Vahl } \\
\text { Scutellaria potosina } \text { Brandegee } \\
\text { Teucrium cubense Jacq. }\end{array}$ & $\begin{array}{l}\text { Menta } \\
\text { Marrubio } \\
\text { Orégano } \\
\text { Mejorana de monte } \\
\text { Salvia de otoño } \\
\text { Salvia de los cañones } \\
\text { Mirto } \\
\text { Salvia de las rocosas } \\
\text { Salvia de la montaña }\end{array}$ & $\begin{array}{c}\text { EA, C } \\
\text { OT } \\
\text { E, EA } \\
\text { MDR, MDM } \\
\text { P, MC, MDR } \\
\text { E } \\
\text { E, EA } \\
\text { E, EA } \\
\text { C, EA, E } \\
\text { EA, MDR } \\
\text { OT } \\
\text { E, EA } \\
\text { E, MC } \\
\text { MDR } \\
\text { MDR, MDM }\end{array}$ \\
\hline LAURACEAE & & \\
\hline Litsea glaucescens Kunth & Laurel & EA, E \\
\hline LOASACEAE & & \\
\hline Mentzelia hispida Willd. & Pegarropa & $\mathrm{E}, \mathrm{P}$ \\
\hline
\end{tabular}


Apéndice. Continuación

\begin{tabular}{|c|c|c|}
\hline Familia y especie & Nombre común & Formación vegetal \\
\hline LOGANIACEAE & & \\
\hline $\begin{array}{l}\text { Buddleja cordata Kunth } \\
\text { Buddleja marrubiifolia Benth. } \\
\text { Buddleja scordioides Kunth }\end{array}$ & $\begin{array}{l}\text { Tepozán } \\
\text { Suelda, escobilla }\end{array}$ & $\begin{array}{c}\text { E, P } \\
\text { MDM, MDR } \\
\text { OT, MDM }\end{array}$ \\
\hline MALVACEAE & & \\
\hline $\begin{array}{l}\text { Anoda cristata (L.) Schltdl. } \\
\text { Malva parviflora L. } \\
\text { Sphaeralcea angustifolia (Cav.) G. Don }\end{array}$ & $\begin{array}{l}\text { Amapolita morada } \\
\text { Malva } \\
\text { Hierba del negro }\end{array}$ & $\begin{array}{l}\text { MDR } \\
\text { MDM, MDR } \\
\text { MDM }\end{array}$ \\
\hline MARTYNIACEAE & & \\
\hline $\begin{array}{l}\text { Proboscidea louisianica ssp. fragrans } \\
\text { (Lindl.) Bretting }\end{array}$ & Toritos & OT \\
\hline MORACEAE & & \\
\hline Morus celtidifolia Kunth & Mora & $E, P$ \\
\hline NYCTAGINACEAE & & \\
\hline $\begin{array}{l}\text { Allionia incarnata L. } \\
\text { Boerhavia anisophylla Torr. } \\
\text { Cyphomeris gypsophiloides (Mart. \& Gal.) } \\
\text { Standl. }\end{array}$ & Corrigüela & $\begin{array}{l}\text { Z, MDM } \\
\text { MDR } \\
\text { Z, MDR }\end{array}$ \\
\hline OLEACEAE & & \\
\hline $\begin{array}{l}\text { Forestiera angustifolia Torr. } \\
\text { Forestiera reticulata Torr. } \\
\text { Fraxinus greggii A. Gray var. greggii } \\
\text { Menodora coulteri A. Gray } \\
\text { Menodora helianthemoides Humb. \& } \\
\text { Bonpl. }\end{array}$ & Panalero & $\begin{array}{l}\mathrm{MC}, \mathrm{MS}, \mathrm{MDR} \\
\mathrm{E} \\
\mathrm{EA}, \mathrm{MDR}, \mathrm{MS} \\
\mathrm{P}, \mathrm{MC}, \mathrm{MDR} \\
\mathrm{MDR}\end{array}$ \\
\hline ONAGRACEAE & & \\
\hline Gaura coccinea (Nutt.) Pursh & & $\mathrm{MC}, \mathrm{MDM}$ \\
\hline
\end{tabular}


Apéndice. Continuación

\begin{tabular}{|c|c|c|}
\hline Familia y especie & Nombre común & Formación vegetal \\
\hline OXALIDACEAE & & \\
\hline $\begin{array}{l}\text { Oxalis albicaulis Kunth } \\
\text { Oxalis corniculata L. }\end{array}$ & $\begin{array}{l}\text { Lujula } \\
\text { Agritos }\end{array}$ & $\begin{array}{l}\mathrm{MC}, \mathrm{EA} \\
\mathrm{EA}\end{array}$ \\
\hline PAPAVERACEAE & & \\
\hline $\begin{array}{l}\text { Argemone fruticosa Thurb. ex A. Gray } \\
\text { Argemone ochroleuca Sweet }\end{array}$ & $\begin{array}{l}\text { Chicalote } \\
\text { Amapola }\end{array}$ & $\begin{array}{l}\text { MDR, E } \\
\text { OT }\end{array}$ \\
\hline PASSIFLORACEAE & & \\
\hline Passiflora tenuiloba Engelm. & & $\mathrm{MDR}, \mathrm{C}$ \\
\hline PHYTOLACCACEAE & & \\
\hline Rivina humilis L. & & MDM \\
\hline PLANTAGINACEAE & & \\
\hline $\begin{array}{l}\text { Plantago nivea Kunth } \\
\text { Plantago linearis Kunth }\end{array}$ & & $\begin{array}{l}\text { P, EA } \\
\text { P, MC }\end{array}$ \\
\hline POLEMONIACEAE & & \\
\hline $\begin{array}{l}\text { Gilia incisa Benth. } \\
\text { Loeselia coerulea (Cav.) G. Don }\end{array}$ & Jarritos & $\begin{array}{c}\mathrm{E} \\
\mathrm{P}, \mathrm{MDR}\end{array}$ \\
\hline POLYGALACEAE & & \\
\hline $\begin{array}{l}\text { Polygala alba Nutt. } \\
\text { Polygala macradenia Gray }\end{array}$ & & $\begin{array}{l}\text { MS, MDR } \\
\text { MDR }\end{array}$ \\
\hline POLYGONACEAE & & \\
\hline $\begin{array}{l}\text { Eriogonum clivosum Hess \& Reveal } \\
\text { Eriogonum viscanum Hess \& Reveal }\end{array}$ & & $\begin{array}{l}\text { OT } \\
\text { OT }\end{array}$ \\
\hline PORTULACACEAE & & \\
\hline $\begin{array}{l}\text { Portulaca pilosa } \mathrm{L} . \\
\text { Portulaca retusa } \text { Engelm. } \\
\text { Talinopsis frutescens A. Gray }\end{array}$ & $\begin{array}{l}\text { Verdolaga } \\
\text { Verdolaga } \\
\text { Agrito }\end{array}$ & $\begin{array}{c}\text { MC, EA } \\
\text { MDR } \\
\text { MDM, MDR }\end{array}$ \\
\hline
\end{tabular}


Apéndice. Continuación

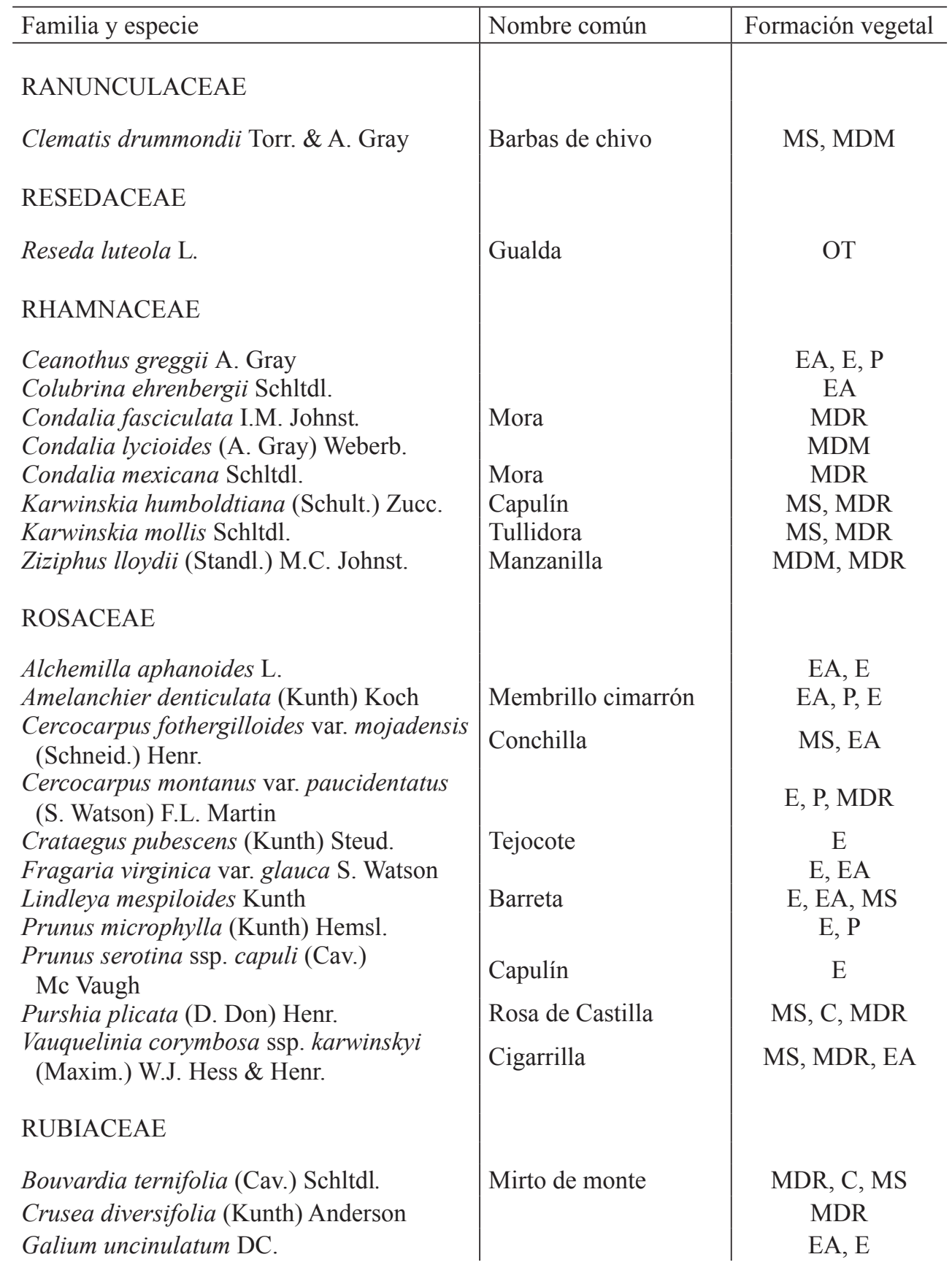


Apéndice. Continuación

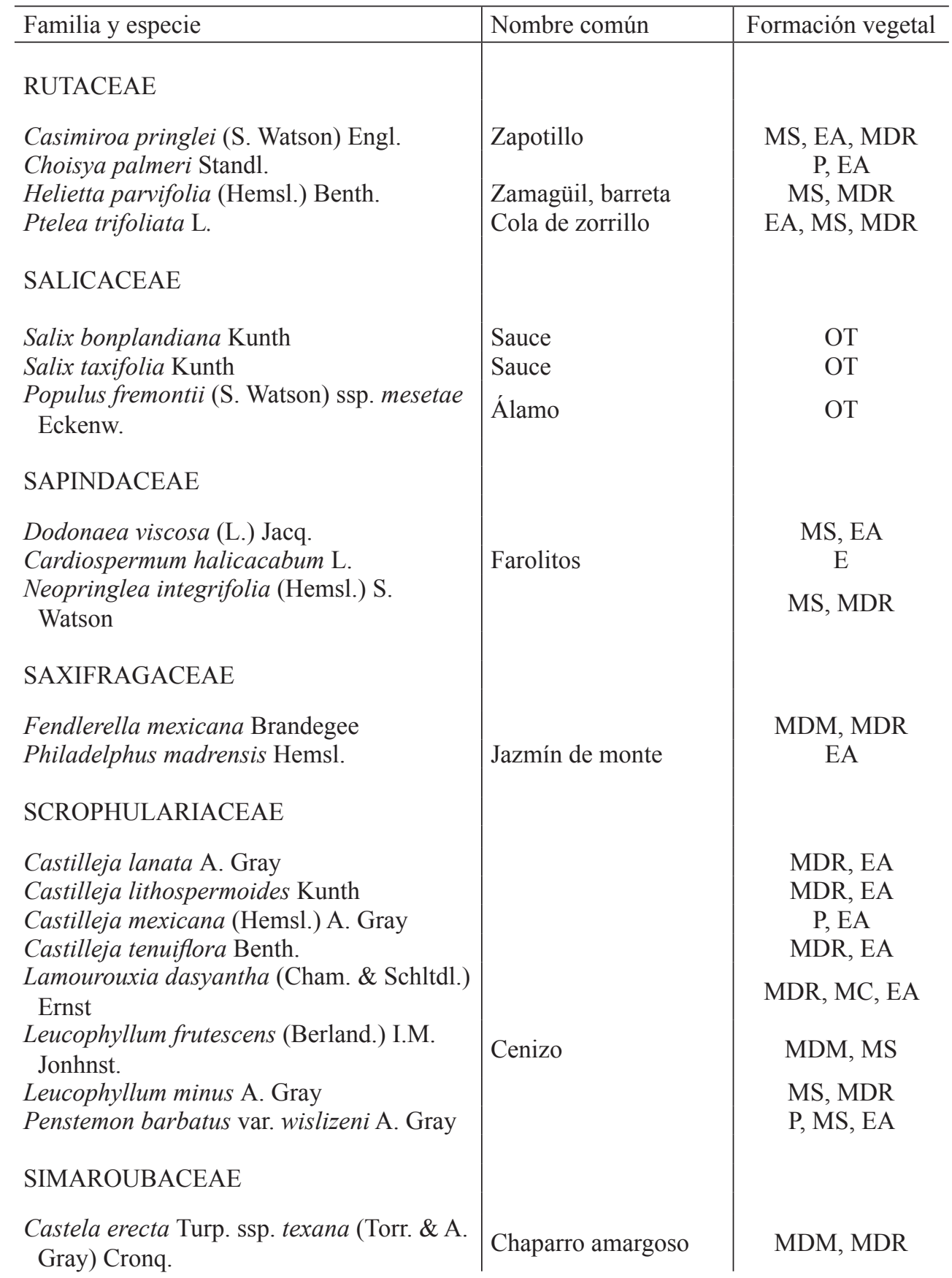


Apéndice. Continuación

\begin{tabular}{|c|c|c|}
\hline Familia y especie & Nombre común & Formación vegetal \\
\hline $\begin{array}{l}\text { Castela stewartii (C.H. Muller) Moran \& } \\
\text { Felger }\end{array}$ & & MDM \\
\hline SMILACACEAE & & \\
\hline Smilax bona-nox L. & & $\mathrm{E}$ \\
\hline SOLANACEAE & & \\
\hline $\begin{array}{l}\text { Datura inoxia Mill. } \\
\text { Datura stramonium L. } \\
\text { Lycium berlandieri Dunal } \\
\text { Nicotiana glauca } \text { Graham } \\
\text { Nicotiana trigonophylla } \text { Dunal } \\
\text { Physalis philadelphica Lam. } \\
\text { Solanum elaeagnifolium Cav. } \\
\text { Solanum nigrescens Mart. \& Gal. } \\
\text { Solanum rostratum } \text { Dunal }\end{array}$ & $\begin{array}{l}\text { Toloache } \\
\text { Toloache } \\
\text { Gigante } \\
\text { Tabaco de coyote } \\
\text { Tomatillo } \\
\text { Trompillo } \\
\text { Mala mujer }\end{array}$ & $\begin{array}{c}\text { OT } \\
\text { OT } \\
\text { MDM } \\
\text { OT } \\
\text { MDM, MDR } \\
\text { OT } \\
\text { OT } \\
\text { OT } \\
\text { OT }\end{array}$ \\
\hline TROPAEOLACEAE & & \\
\hline $\begin{array}{l}\text { Tropaeolum majus L. } \\
\text { ULMACEAE }\end{array}$ & Mastuerzo & OT \\
\hline $\begin{array}{l}\text { Celtis reticulata Torr. } \\
\text { Celtis pallida Torr. }\end{array}$ & $\begin{array}{l}\text { Palo blanco } \\
\text { Granjeno }\end{array}$ & $\begin{array}{l}\text { MDM, Z } \\
\text { MC, MDM }\end{array}$ \\
\hline UMBELIFERAE & & \\
\hline $\begin{array}{l}\text { Eryngium serratum Cav. } \\
\text { URTICACEAE }\end{array}$ & Hierba del gato & OT \\
\hline $\begin{array}{l}\text { Urtica chamaedryoides Pursh } \\
\text { VERBENACEAE }\end{array}$ & Ortiguilla & $\mathrm{MS}, \mathrm{OT}$ \\
\hline $\begin{array}{l}\text { Aloysia lycioides Cham. } \\
\text { Citharexylum brachyanthum (A. Gray) A. } \\
\text { Gray } \\
\text { Citharexylum oleinum (Benth.) Moldenke } \\
\text { Lantana camara L. }\end{array}$ & Mirto de monte & $\begin{array}{c}\text { MDM, MC } \\
\text { MDM, MDR, MS } \\
\text { E, P } \\
\text { MS, MDR }\end{array}$ \\
\hline
\end{tabular}


Apéndice. Continuación




Apéndice. Continuación

\begin{tabular}{|c|c|c|}
\hline Familia y especie & Nombre común & Formación vegetal \\
\hline $\begin{array}{l}\text { Yucca decipiens Trel. } \\
\text { Yucca filifera } \text { Chabaud }\end{array}$ & $\begin{array}{l}\text { Palma } \\
\text { Palma china }\end{array}$ & $\begin{array}{l}\text { MDM MDR } \\
\text { MDM }\end{array}$ \\
\hline ARECACEAE (PALMAE) & & \\
\hline Brahea berlandieri Bartlett & Palmito de mícharo & MS, MDR \\
\hline BROMELIACEAE & & \\
\hline $\begin{array}{l}\text { Hechtia glomerata Zucc. } \\
\text { Tillandsia erubescens Schltdl. } \\
\text { Tillandsia recurvata (L.) L. } \\
\text { Tillandsia usneoides (L.) L. }\end{array}$ & $\begin{array}{l}\text { Guapilla china } \\
\text { Paixtle } \\
\text { Paixtle } \\
\text { Heno }\end{array}$ & $\begin{array}{l}\text { MS, MDR, EA } \\
\text { E, P } \\
\text { MDM } \\
\text { E, P }\end{array}$ \\
\hline COMMELINACEAE & & \\
\hline $\begin{array}{l}\text { Commelina erecta var. angustifolia } \\
\text { (Michx.) Fern. } \\
\text { Gibasis linearis (Benth.) Rohw. } \\
\text { Tradescantia crassifolia var. angustifolia } \\
\text { S. Watson }\end{array}$ & & $\begin{array}{l}\text { MS, MDR, P } \\
\text { MS, MDR } \\
\text { MS, E }\end{array}$ \\
\hline CYPERACEAE & & \\
\hline Carex schiedeana Kuntze & & E, EA \\
\hline LILIACEAE & & \\
\hline $\begin{array}{l}\text { Aloe barbadensis Mill. } \\
\text { Asphodelus fistulosus L. }\end{array}$ & $\begin{array}{l}\text { Sávila } \\
\text { Cebollín }\end{array}$ & $\begin{array}{l}\mathrm{MC}, \mathrm{OT} \\
\mathrm{Z}, \mathrm{EA}\end{array}$ \\
\hline NOLINACEAE & & \\
\hline $\begin{array}{l}\text { Dasylirion acrotriche (Schiede) Zucc. } \\
\text { Dasylirion cedrosanum Trel. } \\
\text { Dasylirion longissimum Lem. } \\
\text { Dasylirion parryanum Trel. } \\
\text { Hesperaloe funifera } \text { (K. Koch) Trel. } \\
\text { Nolina texana S. Watson }\end{array}$ & $\begin{array}{l}\text { Cucharilla } \\
\text { Sotol } \\
\text { Vara de cuete } \\
\text { Sotol } \\
\text { Samandoque } \\
\text { Cortadillo }\end{array}$ & $\begin{array}{l}\text { MDR, MS } \\
\text { MS, MDR } \\
\text { MS, MDR } \\
\text { EA, E }\end{array}$ \\
\hline ORCHIDACEAE & & \\
\hline $\begin{array}{l}\text { Dichromanthus cinnabarinus (Llave \& } \\
\text { Lex.) A. Gray }\end{array}$ & & MDR, MC \\
\hline
\end{tabular}


Apéndice. Continuación

\begin{tabular}{|c|c|c|}
\hline Familia y especie & Nombre común & Formación vegetal \\
\hline \multicolumn{3}{|l|}{ POACEAE (GRAMINEAE) } \\
\hline Agropyron arizonicum Scribn. \& Smith & Zacate azul & E, EA \\
\hline Aristida adscensionis L. & Zacate tres barbas & $\mathrm{Z}, \mathrm{MDR}, \mathrm{EA}$ \\
\hline Aristida barbata E. Fourn. ex Hemsl. & & MDR, EA \\
\hline $\begin{array}{l}\text { Aristida divaricata Humb. \& Bonpl. ex } \\
\text { Willd. }\end{array}$ & & MC,MDM, MDR \\
\hline Aristida schiedeana Trin. \& Rupr. & & MDR, EA \\
\hline Aristida ternipes Cav. & & MDM, MDR \\
\hline Arundo donax L. & Carrizo & OT \\
\hline Bothriochloa barbinodis (Lag.) Herter & & MDM, MC \\
\hline Bouteloua curtipendula (Michx.) Torr. & Zacate banderilla & MDM, MDR, EA \\
\hline Bouteloua chasei Swallen & Zacate navajita salina & $\mathrm{Z}, \mathrm{MDM}$ \\
\hline Bouteloua gracilis (Kunth) Lag. & Zacate navajita azul & Z, MDM, MDR \\
\hline Bouteloua hirsuta Lag. & $\begin{array}{l}\text { Zacate navajita } \\
\text { belluda }\end{array}$ & MDM, MC \\
\hline $\begin{array}{l}\text { Bouteloua scorpioides Lag. } \\
\text { Bouteloua trifida Thurb. }\end{array}$ & Zacate escorpión & $\begin{array}{l}\text { MC, EA, MDR } \\
\text { C, MDR }\end{array}$ \\
\hline $\begin{array}{l}\text { Brachypodium mexicanum (Roem. \& } \\
\text { Schult.) Link }\end{array}$ & & MS, MDR \\
\hline Bromus anomalus Rupr. ex Fourn. & & MS, MDR, MDM \\
\hline Buchloe dactyloides (Nutt.) Engelm. & Zacate búfalo & C, EA \\
\hline Cenchrus incertus M.A. Curtis & Cadillo & OT \\
\hline Chloris gayana Kunth & Zacate rhodes & OT \\
\hline Chloris submutica Kunth & & $\mathrm{P}, \mathrm{MDR}, \mathrm{MC}$ \\
\hline Chloris virgata $\mathrm{S}$. Watson. & Zacate mota & MDR, MC \\
\hline Chondrosum barbatum (Lag.) W.D. Clayton & Pata de cuervo & MDR, MDM \\
\hline Cynodon dactylon (L.) Pers. & Zacate pata de gallo & MDM, OT \\
\hline Echinochloa colona (L.) Link & Zacate de agua & OT, MDR \\
\hline Eleusine indica (L.) Gaertn. & & OT \\
\hline Enneapogon desvauxii P. Beauv. & Zacate ladera & MDR, EA \\
\hline Eragrostis cilianensis Link ex Lutati & Amor seco & MDR \\
\hline Eragrostis intermedia Hitchc. & Zacate pradera & $\mathrm{P}, \mathrm{EA}, \mathrm{MC}$ \\
\hline Eragrostis mexicana (Hornem.) Link & Zacate mexicano & $\mathrm{C}, \mathrm{MDR}$ \\
\hline Erioneuron avenaceum (Kunth) Tateoka & & MDR, EA, MC \\
\hline Erioneuron nealleyi (Vasey) Tateoka & Falso tridente & MDR, MS, MDM \\
\hline Erioneuron pulchellum (Kunth) Tateoka & Zacate pelechillo & MDR, MS \\
\hline Heteropogon contortus (L.) P. Beauv. & Zacate colorado & MDM, OT \\
\hline Hilaria cenchroides Kunth & Zacate toboso & $\mathrm{C}, \mathrm{Z}$ \\
\hline Lycurus phleoides Kunth & Zacate lobero & $\mathrm{EA}, \mathrm{C}, \mathrm{Z}$ \\
\hline Microchloa kunthii Desv. & Zacate hoz & EA, MDR, MC \\
\hline
\end{tabular}


Apéndice. Continuación

\begin{tabular}{|c|c|c|}
\hline Familia y especie & Nombre común & Formación vegetal \\
\hline $\begin{array}{l}\text { Muhlenbergia distans Swallen } \\
\text { Muhlenbergia dubia Fourn. } \\
\text { Muhlenbergia firma Beal } \\
\text { Muhlenbergia glauca (Nees) Mez } \\
\text { Muhlenbergia gypsophila C. Reeder \& } \\
\text { Reeder } \\
\text { Muhlenbergia microsperma (DC.) Trin. } \\
\text { Muhlenbergia minutissima (Steud.) Swallen } \\
\text { Muhlenbergia pubescens (Kunth) Hitchc. } \\
\text { Muhlenbergia purpusii Mez } \\
\text { Muhlenbergia repens (Presl) Hitchc. } \\
\text { Muhlenbergia rigida (Kunth) Trin. } \\
\text { Muhlenbergia villiflora Hitchc. } \\
\text { Nassella leucotricha (Trin. \& Rupr.) Pohl } \\
\text { Nassella mucronata (Kunth) Pohl } \\
\text { Nassella tennuissima (Trin.) Barkworth } \\
\text { Panicum obtusum Kunth } \\
\text { Pappophorum bicolor Fourn. } \\
\text { Pennisetum ciliare (L.) Link } \\
\text { Piptochaetium brevicalyx (Fourn.) Ricker } \\
\text { Piptochaetium fimbriatum (Kunth) Hitchc. } \\
\text { Rhynchelytrum repens (Willd.) C.E. Hubb. } \\
\text { Scleropogon brevifolius Phil. } \\
\text { Setaria geniculata (Lam.) Beauv. } \\
\text { Setaria grisebachii Fourn. } \\
\text { Setaria leucopila (Scribn. \& Merr.) K. } \\
\text { Schum. } \\
\text { Setaria macrostachya Kunth } \\
\text { Sorghastrum nutans (L.) Nash } \\
\text { Sporobulus airoides (Torr.) Torr. } \\
\text { Stipa eminens Cav. } \\
\text { Stipa ichu (Ruiz \& Pavón) Kunth } \\
\text { Tragus berteronianus Schult. } \\
\text { Tridens grandiflorus (Vasey) Wooton \& } \\
\text { Standl. } \\
\text { Zea mays L. }\end{array}$ & $\begin{array}{l}\text { Zacate pajita } \\
\text { Zacate espiga } \\
\text { Zacate tempranero } \\
\text { Zacate alcalino } \\
\text { Zacate flechilla } \\
\text { Zacatón } \\
\text { Tridente } \\
\text { Maíz }\end{array}$ & $\begin{array}{c}\text { E, MDR } \\
\text { P, EA, MDR } \\
\text { EA, E, P } \\
\text { P, E, EA } \\
\text { Z, MDM } \\
\text { EA, Z } \\
\text { Z, E, P } \\
\text { P, E, MDR } \\
\text { MDM, Z } \\
\text { Z, MDM } \\
\text { MDR, MC, P } \\
\text { Z, MDM } \\
\text { EA, MDR } \\
\text { E, EA } \\
\text { E, P, MDR } \\
\text { OT } \\
\text { E, P } \\
\text { OT } \\
\text { Z, MDR, EA } \\
\text { E, EA, P } \\
\text { E, P, EA } \\
\text { MDR, MC } \\
\text { C, MDR } \\
\text { C, MDR } \\
\text { MS, MDR } \\
\text { E, C, MDR } \\
\text { E, C, EA } \\
\text { MDM, MDR, Z } \\
\text { Z, E, MS } \\
\text { EA, E, Z } \\
\text { Z, MDR } \\
\text { MDM, MDR } \\
\text { OT }\end{array}$ \\
\hline
\end{tabular}

